\title{
Reelin reverts biochemical, physiological and cognitive alterations in mouse models of Tauopathy
}

\author{
Daniela Rossi $^{\mathrm{a}, \mathrm{b}, \mathrm{c}}$, Agnès Gruart ${ }^{\mathrm{d}}$, Gerardo Contreras-Murillo ${ }^{\mathrm{d}}$, Ashraf Muhaisen ${ }^{\mathrm{a}, \mathrm{b}, \mathrm{c}}$, Jesús Ávila ${ }^{\mathrm{e}}$, \\ José María Delgado-García ${ }^{\mathrm{d}}$, Lluís Pujadas ${ }^{\mathrm{a}, \mathrm{b}, \mathrm{c}, *, 1}$, Eduardo Soriano ${ }^{\mathrm{a}, \mathrm{b}, \mathrm{c}, \mathrm{f}, *, 1}$ \\ a Vall d'Hebron Institut de Recerca, 08035, Barcelona, Spain \\ ${ }^{\mathrm{b}}$ Department of Cell Biology, Physiology and Immunology, and Institute of Neurosciences, University of Barcelona, 08028, Barcelona, Spain

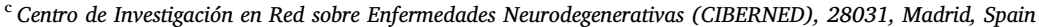 \\ ${ }^{\mathrm{d}}$ Division of Neurosciences, Universidad Pablo de Olavide, 41013, Sevilla, Spain \\ e Centro de Biología Molecular Severo Ochoa, 28049, Madrid, Spain \\ f ICREA Academia, 08010, Barcelona, Spain
}

\section{A R T I C L E I N F O}

\section{Keywords:}

Intracellular signaling

Reelin

Tauopathy

Electrophysiology

Cognition

Alzheimer's disease

\begin{abstract}
A B S T R A C T
Reelin is an extracellular protein crucial for adult brain plasticity. Moreover, Reelin is protective against amyloid- $\beta$ (A $\beta$ ) pathology in Alzheimer's Disease (AD), reducing plaque deposition, synaptic loss and cognitive decline. Given that Tau protein plays a key role in $\mathrm{AD}$ pathogenesis, and that the Reelin pathway modulates Tau phosphorylation, here we explored the involvement of Reelin in AD-related Tau pathology. We found that Reelin overexpression modulates the levels of Tau phosphorylation in AD-related epitopes in VLW mice expressing human mutant Tau. in vitro, Reelin reduced the A $\beta$-induced missorting of axonal Tau and neurofilament proteins to dendrites. Reelin also reverted in vivo the toxic somatodendritic localization of phosphorylated Tau. Finally, overexpression of Reelin in VLW mice improved long-term potentiation and long-term memory cognitive performance thus masking the cognitive and physiological deficits in VLW mice. These data suggest that the Reelin pathway, which is also protective against $\mathrm{A} \beta$ pathology, modulates fundamental traits of Tau pathology, strengthening the potential of Reelin as a therapeutic target in $\mathrm{AD}$.
\end{abstract}

\section{Introduction}

Reelin is an extracellular matrix protein that controls neuronal migration in the embryonic brain, being a major actor in corticogenesis and in the lamination of other brain areas, such as the cerebellum (D'Arcangelo and Reeler, 1998; Rice and Curran, 2001; Soriano and Del Rio, 2005). Reelin is also expressed in the adult brain (Alcantara et al., 1998), where it regulates the induction of synaptic plasticity (Herz and Chen, 2006) and potentiates glutamatergic neurotransmission, directly regulating $N$-methyl-D-aspartate (NMDA) receptor subunit trafficking and function (Durakoglugil et al., 2009; Groc et al., 2007). The overexpression of Reelin in the adult mouse brain (TgRln model) drives an increase in excitatory synaptic contacts, hypertrophy of dendritic spines, and enhanced long-term potentiation (LTP) responses (Pujadas et al., 2010). These observations thus indicate that Reelin is potentially involved in the modulation of cognition, learning and memory.
The homeostasis of key players underlying synaptic plasticity is fundamental for the maintenance of cognitive functions in the adult brain and for implications in neurodegenerative diseases. Alzheimer's disease (AD), the most prevalent form of dementia worldwide (Reitz and Mayeux, 2014), is characterized by abnormal dendritic spine morphology and density (Spires-Jones and Knafo, 2012) and by synaptic dysfunction (Sivanesan et al., 2013). Indeed, synaptic loss shows a greater correlation with cognitive impairment than histopathological hallmarks of the disease such as amyloid plaques, thus leading to consider AD as a "synaptic failure"-disease (Selkoe, 2002). Of note, both in the J20 AD mouse model (carrying the Swedish and the Indiana mutations of the human Amyloid Precursor Protein) and in AD patients, depletion of Reelin has been reported as one of the early events in this pathology (Herring et al., 2012; Chin et al., 2007). Consistently, overexpression of Reelin in the $\mathrm{J} 20 \mathrm{AD}$ model reduces not only amyloid plaque deposition but also synaptic loss, while rescuing memory-

\footnotetext{
* Corresponding authors at: Department of Cell Biology, Physiology and Immunology, and Institute of Neurosciences, University of Barcelona, 08028, Barcelona, Spain.

E-mail addresses: 1luis.pujadas@ub.edu (L. Pujadas), esoriano@ub.edu (E. Soriano).

${ }^{1} \mathrm{LP}$ and ES are co-senior authors.
} 
associated cognitive impairment (Pujadas et al., 2014).

Although $A \beta$ oligomers have been widely recognized as the main species responsible for synaptic dysfunction in AD (Selkoe, 2008; Walsh et al., 2002; Haass and Selkoe, 2007), recent data indicate that their toxicity is mediated by Tau protein (Ittner and Gotz, 2011; Ittner et al., 2010; Bloom, 2014), whose intracellular aggregates, known as neurofibrillary tangles (NFTs), are another hallmark of the disease. In addition, Tau pathology in the human brain has a high correlation with brain atrophy and severe cognitive impairment (Jagust, 2018).Together with MAP1 and MAP2, Tau is one of the major brain-specific microtubule-associated proteins (MAPs) involved in the stabilization of the microtubule network, among other physiological functions (Wang and Mandelkow, 2016). Tau develops its brain-specific functions as an axonal protein, and its activity is regulated by its degree of phosphorylation at different tyrosine, threonine or serine residues. In the $\mathrm{AD}$ brain, phosphorylated Tau (phospho-Tau) levels are three- to four-fold higher than in the healthy brain, and Tau starts to be phosphorylated at abnormal sites (Kopke et al., 1993; Iqbal et al., 2010). In this "hyperphosphorylated" state, Tau detaches from microtubules and mislocalizes to the somatodendritic compartment, where it polymerizes into paired helical filaments (PHFs), finally resulting in the formation of NFTs (Wang and Mandelkow, 2016; Ballatore et al., 2007). Moreover, when abnormally modified, Tau becomes enriched in dendritic spines and thus interferes with neurotransmission (Hoover et al., 2010). A $\beta$ oligomers promote both Tau hyperphosphorylation and its mislocalization to dendrites and postsynaptic enrichment, finally causing depletion of dendritic spines (Zempel et al., 2013; Zempel et al., 2010).

In the context of $\mathrm{AD}$ pathology, in addition to interacting with and reducing the toxicity of $A \beta$ species (Pujadas et al., 2014), Reelin signaling triggers an intracellular cascade in which AKT kinase is induced. This activation finally leads to the modulation of the activity of GSK-3 $\beta$, the major kinase for Tau protein (Gonzalez-Billault et al., 2005; Beffert et al., 2002). Indeed, mutant mice deficient in Reelin, in its transducer Dab1, or in Reelin receptors ApoER2 and/or VLDLR show increased levels of Tau phosphorylation (Hiesberger et al., 1999; Ohkubo et al., 2003). Of note, the APOE4 protein, which binds APOER2 receptors, has recently been found to exacerbate Tau pathology (Shi et al., 2017).

Moreover, Reelin haploinsufficiency (heterozygous reeler) in $\mathrm{AD}$ mice results in accelerated AD-like pathology, enhancing both amyloid plaque burden and NFT deposition (Kocherhans et al., 2010), while adult conditional Reelin knock-out mice display severe memory impairment when exposed to very low amounts of amyloid deposition (Lane-Donovan et al., 2015). Conversely, whether the overactivation of Reelin signaling is able to reverse Tau hyperphosphorylation in the context of $\mathrm{AD}$ has not been explored to date.

Here we examined whether transgenic Reelin overexpression in an $\mathrm{AD}$ background alters pathological levels of phospho-Tau and ameliorates the cognitive deficits associated with Tau pathology. We show that Reelin overexpression modulates in vivo the levels of Tau phosphorylation at some AD-related epitopes in the VLW Tauopathy mouse model (Lim et al., 2001). Concomitantly, both in vitro and in vivo, Reelin reduced the toxicity-associated somatodendritic localization of NF and phospho-Tau. Finally, VLW mice showed improved LTP responses and cognitive performance in the passive avoidance paradigm upon Reelin overexpression. Taken together, our results complement the previously reported beneficial effect of Reelin in AD pathology and confer the Reelin pathway a pivotal role as a negative regulator of $\mathrm{AD}$ progression, by antagonizing both $A \beta$ and Tau pathologies.

\section{Material and methods}

\subsection{Animals}

The TgRln (Tg1/Tg2; pCamKII-tTA/tetO-rlM) mouse is a conditional regulated double transgenic line based on a Tet-off regulated binary system that achieves Reelin overexpression under the control of calcium/calmodulin-dependent kinase II $\alpha$ promoter (pCaMKII $\alpha$ ) (Pujadas et al., 2010). The GSK-3 $\beta$ (Tg1/Tg3; pCaMKII-tTA/BitetO $\beta$ Gal/GSK-3 $\beta$ ) mouse is a conditional regulated double transgenic line showing GSK- $3 \beta$ protein overexpression (and the reporter gene $\beta-\mathrm{Gal}$ ) also under the control of pCaMKIIa (Lucas et al., 2001). The VLW (Tg4; pThy1-Tau-VLW) mouse is a constitutive transgenic line expressing human Tau protein with four tubulin-binding repeats (increased by FTDP-17 splice donor mutations) and three FTDP-17 missense mutations: G272 V, P301 L, and R406W under the control of thy 1 promoter gene cassette (Lim et al., 2001). TgRln/GSK-3 $\beta$ (Tg1/Tg2/Tg3) and TgRln/VLW (Tg1/Tg2/Tg4) mice are triple transgenic, obtained by interbreeding the previously described strains. Control animals used throughout the study were littermates of transgenics not bearing overexpression of Reelin, GSK-3ß, or VLW (i.e. wild-types, single carriers of $\mathrm{Tg} 1, \mathrm{Tg} 2$ or $\mathrm{Tg} 3$ transgenes, or double $\mathrm{Tg} 2 / \mathrm{Tg} 3$ ). All the transgenic animals used in this study are kept in homozygosis for each transgene. Both male and female animals were used, thus representing the diversity of the population in biochemical and histological experiments; only males were used in behavioral and electrophysiological experiments due to the well-known variability in such tests. Mice were bred, studied and processed in the animal research facility at the Faculty of Pharmacy of the University of Barcelona or sent to the animal research facility of the Pablo de Olavide University for behavioral studies. Animals were provided with food and water ad libitum and maintained in a temperature-controlled environment in a $12 / 12 \mathrm{~h}$ lightdark cycle. All the experiments involving animals were performed in accordance with the European Community Council directive and the National Institute of Health guidelines for the care and use of laboratory animals. Experiments were also approved by the local ethical committees.

\subsection{Chemicals}

Diaminobenzidine reagent (DAB), Hydrogen Peroxide $\left(\mathrm{H}_{2} \mathrm{O}_{2}\right)$, 4'-6'Diamino-2-Phenylindole (DAPI), Triton X-100, Dimethyl Sulfoxide (DMSO), Nissl (Cresyl violet acetate and Thionine acetate salt), Hexadeuterodimethyl sulfoxide (DMS $=-\mathrm{d} 6$ ), magnesium chloride, HEPES buffer, EGTA, 1,1,1,3,3,3-Hexafluoro-2-propanol and Phalloidin-Tetramethylrhodamine $\mathrm{B}$ isothiocyanate (red-phalloidin) were from Sigma. Paraformaldehyde (PF), Eukitt mounting medium, Glycerol, Ethylene Glycol, Phosphate Buffe TRIS buffer, sodium chloride, sodium orthovanadate, sodium fluoride and gelatine were from Panreac. Mowiol 4-88 reagent was from Calbiochem. Nickel ammonium sulfate was from Analyticals. Tetra-sodium pyrophosphate was from Fluka. Glycerol was from Merck. Protein A and Protein G Sepharose 4 Fast Flow were from GE Healthcare.

\subsection{Antibodies}

Mouse anti-phopsho-Tau (ser 396/404) PHF-1 was a kind gift from Dr. Peter Davies, Albert Einstein College of Medicine, New York, NY (Greenberg et al., 1992). The following commercial primary antibodies were used: anti-Reelin (clone G10) (Chemicon); anti-phospho-Tau (ser202/thr205, clone AT8, Innogenetics); anti-total Tau (clone Tau-5, Millipore); anti-total Tau K9JA (DAKO, A0024); anti-actin (Chemicon, MAB1501); anti-phospho-Tau T205 (ThermoFisher, 44-738G); antineurofilament (NF200, Sigma, N4142); anti-calbindin (Swant, CB38); anti-MAP2 (Sigma, M1406); anti-human Tau HT7 (Thermofisher, MN1000); and anti- $\beta$ Galactosidase (AB986, Millipore). The HRP-labeled secondary antibodies used for western blot were from DAKO. Biotinylated-secondary antibodies and Streptavidin-biotinylated/HRP complex were from GE Healthcare. F(ab')2 fragment anti-mouse IgG was supplied by Jackson Immuno Research. The fluorescent secondary antibodies used for immunofluorescence were purchased from Invitrogen (Alexafluor). 


\subsection{Software}

NDP.view2 (Hamamatsu Photonics) was used to magnify areas of interest from Nanozoomer-scanned immunohistochemical slides. Image $\mathrm{J}$ was used as a general tool for the analysis, measuring, processing and quantification of images. Gel-Pro Analyzer was used for the quantification of WB-scanned films. GraphPad Prism was used for graphical representation and statistical analysis of the data.

\subsection{A $\beta$-derived diffusible ligands (ADDLs) preparation}

ADDLs were prepared as described (Pujadas et al., 2014; Lambert et al., 2001). Briefly, $A \beta_{42}$ was dissolved in hexafluoro-2-propanol $(1 \mathrm{mg} / \mathrm{ml})$ and aliquoted in low-binding Eppendorf tubes. Hexafluoro2-propanol was then removed by freeze-drying. An aliquot of $A \beta_{42}$ was dissolved in anhydrous DMSO-d6 to $5 \mathrm{mM}$ and further diluted with icecold F12 medium (GIBCO) without phenol red to $100 \mathrm{~mm}$. This solution was incubated at $4{ }^{\circ} \mathrm{C}$ for $24 \mathrm{~h}$ and then centrifuged at $14000 \mathrm{~g}$ for 10 min. The $A \beta_{42}$ concentration in the supernatant was determined using the Bradford assay and found to range between 60 and $100 \mathrm{mM}$. The peptide solution was diluted to the desired concentrations for primary hippocampal cell treatments $(1$ and $3 \mu \mathrm{M})$.

\subsection{Primary hippocampal cultures}

Hippocampal neurons were obtained from E16 CD1 mouse embryos (animal research facility at the Faculty of Pharmacy of the University of Barcelona). Brains were dissected in PBS containing $0.6 \%$ glucose, and hippocampi were excised. After trypsin (GIBCO) and DNAse (Roche Diagnostics) treatments, hippocampi were dissociated by gentle sweeping. Cells were counted and seeded at $3 \times 10^{4}$ cells per well in four-well plates onto poly-D-lysine-coated coverslips in Neurobasal medium containing B27 supplement (GIBCO) supplemented with GlutaMAX (GIBCO), Penicillin-Streptomicin (GIBCO) and 1:5 of conditioned media obtained from astrocyte cultures. Neuronal cultures were kept for 21 days in vitro (DiV) in $5 \% \mathrm{CO} 2$ at $37^{\circ} \mathrm{C}$ before treatment. Cells were incubated for $3 \mathrm{~h}$ at $37^{\circ} \mathrm{C}$ with ADDLs $(1$ and $3 \mu \mathrm{M})$ or corresponding volumes of vehicle ( $0.1 \%$ DMSO in F12 medium), with or without the addition of $5 \mu \mathrm{g} / \mathrm{ml}$ Reelin (or equivalent volumes of Mock). Addition of vehicle/ADDLs and Mock/Reelin was made sequentially during treatments, without mixture of the two agents outside the culture media. Cells were then washed 3 times with phosphate buffer saline (PBS) and fixed for 20 min with $4 \%$ paraformaldehyde (PF) in $0.1 \mathrm{M}$ phosphate buffer (PB). For immunofluorescence, cells were blocked for $2 \mathrm{~h}$ at room temperature (RT) with PBS containing 10 $\%$ of normal goat serum (NGS) and $0.1 \%$ Triton in $0.2 \%$ of gelatin. Primary antibodies (NF200 1:1000; K9JA 1:50000; MAP2 1:500) were incubated overnight at $4{ }^{\circ} \mathrm{C}$. Incubation with fluorescent secondary antibody $(1: 200 ; 2 \mathrm{~h}$ at $\mathrm{RT})$, red phalloidin $(2 \mu \mathrm{g} / \mathrm{mL})$ and DAPI $(0.02 \mu \mathrm{g} / \mathrm{mL})$ was performed in PBS-5 \% NGS. Coverslips were mounted in Mowiol.

\subsection{Fluorescence imaging and detection of missorting}

Hippocampal neurons were observed with a $40 \mathrm{X}$ objective on an Olympus CellR/ScanR microscope. Micrograph mosaics of $10 \times 10$ images were randomly taken from coverslips for each condition. Fields covering an area of $2.9-3 \mathrm{~mm}^{2}$ and containing a range from 30 to 300 neurons were quantified. Tau and NF200 missorting was determined by counting the number of neurons displaying at least one dendrite with increased signal versus the total number of neurons per mosaic/field in a semi-automated manner when possible. NF200 missorting quantification was performed by implementing a macro for automate detection of candidate-neurons with missorted signal; briefly, cells in the culture were detected using cell counting upon nuclei marker (blue, DAPI staining), then compared using a colocalization mask with neuronal marker (red, MAP2 staining) to identify neurons and again compared using a colocalization mask with abnormal axonal somatic staining (green, NF200 staining) to identify candidate-neurons with missorted signal; those candidate cells were finally confirmed by individual observation in double blind experiment to determine neurons with missorted NF200. Tau missorting quantification was performed by counting in each field the total number of neurons and those with missorted phenotype in double blind experiments; neurons in the culture were individually identified considering cell morphology (redPhalloidin staining) and Tau content (green, Tau K9JA staining) and neurons with missorted signal were individually identified considering Tau distribution inside the cell.

Images obtained from ScanR microscopy were quantified along the proximal $20 \mu \mathrm{m}$ of primary dendrites in both translocated and untranslocated dendrites. Intensities for NF200- and Tau-signaling were determined in all conditions by performing a plot-profile along translocated dendrites from cell soma in $2 \mu \mathrm{m}$ thickness. Untreated untranslocated dendrite intensities were used in each experiment for normalization. Quantitative data was obtained from 3 to 4 independent experiments (each including all treatment conditions). Each experiment included 2-3 replicates per condition. Each independent experiment was processed and quantified in bulk.

\subsection{Western blot}

To obtain protein extracts from brain tissues, animals were killed by dislocation and brains were immediately removed. Specific brain regions were rapidly dissected, frozen in liquid nitrogen, and stored at $-80^{\circ} \mathrm{C}$ before processing. Frozen pieces of tissues were also lysed using power homogenizer (Polytron) in 10 volumes of lysis buffer $(50 \mathrm{mM}$ HEPES, pH 7.5, $150 \mathrm{mM}$ sodium chloride, $1.5 \mathrm{mM}$ magnesium chloride, 1 mM EGTA, $10 \%$ glycerol, and $1 \%$ Triton X-100) containing Complete Mini protease inhibitor cocktail (Roche) and phosphatase inhibitors (10 mM tetra-sodium pyrophosphate, $200 \mu \mathrm{M}$ sodium orthovanadate, and $10 \mathrm{mM}$ sodium fluoride).

Samples were sonicated, insoluble debris was removed by centrifugation ( $15 \mathrm{~min}, 16,000 \mathrm{X} \mathrm{g}$ ), and supernatants were stored at $-80^{\circ} \mathrm{C}$. A preclearing step was included when needed for removal of endogenous immunoglobulins by incubating samples with $30 \mu \mathrm{L}$ of Protein-G beads for $90 \mathrm{~min}$ at $4{ }^{\circ} \mathrm{C}$ then recovering cleaned supernatants by centrifugation. Samples were diluted 1:6 with $6 \mathrm{X}$ loading buffer (0.5 M Tris-HCl, pH 6.8, 2.15 M $\beta$-mercaptoethanol, $10 \%$ SDS, $30 \%$ glycerol, and $0.0 .2 \%$ bromophenol blue), boiled for $3 \mathrm{~min}$ at $95^{\circ} \mathrm{C}$. Samples were resolved by SDS-8 \% polyacrylamide gels and transferred onto nitrocellulose membranes. Membranes were then blocked for $1 \mathrm{~h}$

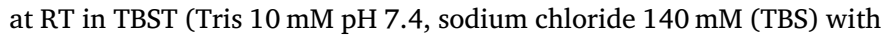
$0.1 \%$ Tween 20) containing $5 \%$ non-fat milk or $3 \%$ BSA. Primary antibodies were incubated for $90 \mathrm{~min}$ in TBST-0.02 \% azide (anti-actin 1:100,000; PHF-1 1:500; AT8 1:500; T205 1:1000; total Tau K9JA 1:20,000; or total Tau5 1:1000). After incubation with anti-mouse or anti-rabbit secondary HRP-labeled antibodies for $1 \mathrm{~h}$ at RT (diluted 1:2000 in TBST-5\% non-fat milk), membranes were developed with the ECL system (GE Healthcare).

\subsection{Histology}

Animals were anesthetized and perfused for 20 min with $0.1 \mathrm{M} \mathrm{PB}$ containing $4 \%$ of $\mathrm{PF}$. Brains were removed, postfixed overnight with PB-4 \% PF, cryoprotected with PB-30 \% sucrose and frozen. Brains were sectioned coronally at $30 \mu \mathrm{m}$ in a cryostat and maintained at $-20^{\circ} \mathrm{C}$ in PB-30 \% glycerol-30 \% ethylene glycol. For immunodetection of antigens, sections were incubated with $10 \%$ methanol- $3 \% \mathrm{H}_{2} \mathrm{O}_{2}$ and then blocked for $2 \mathrm{~h}$ at RT with PBS containing $10 \%$ of either normal goat serum (NGS) or normal horse serum (NHS), $0.2 \%$ of gelatine, $0.3 \%$ Triton and $\mathrm{F}\left(\mathrm{ab}^{\prime}\right) 2$ fragment anti-mouse IgG (1:300) when needed. Primary antibodies (PHF-1 1:150; T205 1:300; calbindin 1:3000; HT7 
1:500; anti- $\beta$ Galactosidase 1:200; anti-Reelin 1:200) were incubated overnight at $4{ }^{\circ} \mathrm{C}$ with PBS-5\% NGS or $-5 \%$ NHS. For immunohistochemistry, sequential incubation with biotinylated secondary antibody (1:200; $2 \mathrm{~h}$ at RT) and streptavidin-HRP $(1: 400 ; 2 \mathrm{~h}$ at RT) was performed in PBS-5 \% NGS or $-5 \%$ NHS. Bound antibodies were visualized by reaction using $\mathrm{DAB}$ and $\mathrm{H}_{2} \mathrm{O}_{2}$ as peroxidase substrates, adding nickel ammonium sulfate in the solution in some cases. Sections were dehydrated, and mounted (Eukitt). For each immunostaining, we developed in bulk the tissue sections from the different genotypes, for all the steps of the process including processing, staining, imaging and analysis. To evaluate the immunohistochemical phospho-Tau signal we quantified the area occupied with positive staining in each of the areas of interest (i.e. CA1 stratum radiatum and granular layer for the VLW strain; and CA3 stratum lucidum for the GSK-3 $\beta$ strain). Images obtained from Nanozoomer scans at $20 \mathrm{X}$ were enlarged to the required magnification with NDPview2 software and processed with ImageJ in a double-blind analysis as follows: first, the area to be analyzed was delimited on 8-bit converted images; second, thresholds of positive/negative signal were determined in areas without staining in the same preparations; third, thresholds were applied to generate binary images; finally, the percentage of the area of interest occupied with positive signal was calculated on binary images.

\subsection{Behavioral analysis}

\subsubsection{Open field}

The open field test is a behavioral motor task aimed at determining the general locomotor activity of experimental animals, their willingness to explore new environments and their anxiety levels. Experiments were carried out in an open field (a box of $28 \times 28 \times 21 \mathrm{~cm}$ ) apparatus (Actifot 809 from Cibertec S.A., Madrid, Spain). Mice (control = 10; TgRln $=8 ; \mathrm{VLW}=15 ; \mathrm{TgRln} / \mathrm{VLW}=10$ ) were placed in the center of the arena and observed for $15 \mathrm{~min}$. The apparatus was provided with infrared lights, located every $2 \mathrm{~cm}$, in the three $(\mathrm{X}, \mathrm{Y}, \mathrm{Z})$ spatial axes. Animals' movements in the arena were quantified automatically with the help of a computer program (MUX_XYZ16L), also from Cibertec S.A. The computer program also allowed us to discriminate when the animal was located at the center $(17 \times 17 \mathrm{~cm})$ or at the periphery (the surrounding area) of the arena. The apparatus was located in a sound-proof room and the experimental area was homogeneously dimly illuminated. The whole apparatus was carefully cleaned with alcohol $\left(70^{\circ}\right.$ proof) after each use. Total mobility in the $\mathrm{X}, \mathrm{Y}$, and $\mathrm{Y}$ (i.e., rearing) axes was quantified in arbitrary units, as was the total time (in min) spent in the center and the periphery of the arena.

\subsubsection{Elevated path test}

This test was designed to check the motor ability of the animals. The elevated path consisted of a $40 \mathrm{~cm}$ long, $5 \mathrm{~cm}$ wide bar located $60 \mathrm{~cm}$ over a soft cushion. Each mouse (control = 19; $\mathrm{TgRln}=13$; $\mathrm{VLW}=10$; $\mathrm{TgRln} / \mathrm{VLW}=14$ ) was placed in the center of the elevated bar and allowed a maximum of $30 \mathrm{~s}$ to reach one of the platforms $(12 \times 12 \mathrm{~cm})$ located at each end of the bar. We quantified latency to fall (in s) (test 1). The test was repeated $24 \mathrm{~h}$ later (test 2).

\subsubsection{Rotarod}

The rotarod test is a behavioral task that assesses motor coordination performance. In this study, we used an accelerating rotarod treadmill (Ugo Basile, Varese, Italy). Mice (control $=13$; TgRln $=9$; $\mathrm{VLW}=10 ; \mathrm{TgRln} / \mathrm{VLW}=14$ ) were placed on the rod and tested at 2-20 rpm (of increasing speed) the first day, for a maximum of $300 \mathrm{~s}$ at each speed. Between trials, mice were allowed to recover in their cages. The total time that each animal remained on the rod was computed as latency to fall, recorded automatically by a trip switch under the floor of each rotating drum. Mice were tested two times with an interval of one hour during the same session. Animals were re-tested 24 and $72 \mathrm{~h}$ later. Results were evaluated by averaging the data from the 4 trials corresponding to sessions 2 and 3 .

\subsubsection{Passive avoidance test}

In this case, mice (control $=19 ; \mathrm{TgRln}=13$; VLW $=9$; TgRln/ $\mathrm{VLW}=14$ ) were placed in darkness for $5 \mathrm{~min}$ before training. They were then placed individually in an illuminated box $(10 \times 13 \times 15 \mathrm{~cm})$ connected to a dark box of the same size. The dark box was equipped with an electric grid floor and separated by an automatic door (passive avoidance device, Ugo Basile, Comerio, VA, Italy). This door was opened $60 \mathrm{~s}$ later. Entry of animals into the dark box was punished by a timed electric foot-shock $(0.5 \mathrm{~mA}, 1 \mathrm{~s})$. After $24 \mathrm{~h}$, pre-trained animals were placed in the illuminated box again and observed for $3 \mathrm{~min}$. The time that the mice took to enter the dark box was noted, and the mean time was calculated for each experimental group. Animals were retested $24 \mathrm{~h}$ later. Latency (in s) was determined automatically by the experimental device (Eleore et al., 2007).

\subsection{Electrophysiological recordings}

Animals were anesthetized with $0.8-1.5 \%$ isofluorane (Astra Zeneca, Madrid, Spain) delivered from a calibrated mask (Cibertec, Madrid, Spain). Animals were chronically implanted with bipolar stimulating electrodes in the right (contralateral) Schaffer collateral-commissural pathway of the dorsal hippocampus ( $2 \mathrm{~mm}$ lateral and $1.5 \mathrm{~mm}$ posterior to bregma, and $1.0-1.5 \mathrm{~mm}$ below brain surface; Paxinos and Franklin, 2004) and with a recording electrode in the ipsilateral stratum radiatum underneath the CA1 area $(1.2 \mathrm{~mm}$ lateral and $2.2 \mathrm{~mm}$ posterior to bregma, and $1.0-1.5 \mathrm{~mm}$ below brain surface). Electrodes were made of $50 \mathrm{~m}$, Teflon-coated tungsten wire (Advent Research Materials, Eynsham, UK). The final location of the recording electrode in was determined following the field potential depth profile evoked in the CA1 area by paired (40-ms interval) pulses presented to the ipsilateral Schaffer collateral pathway (Gruart et al., 2006). The recording electrode was fixed at the site where a reliable monosynaptic fEPSP was recorded. Two bare silver wires $(0.1 \mathrm{~mm}$ in diamater) were affixed to the skull as ground. Electrodes were connected to a 6-pin socket (RS-Amidata, Madrid, Spain). The socket was fixed to the skull with the help of three small screws and dental cement. Further details of this chronic preparation has been described elsewere (Gruart et al., 2006). Animals were allowed a week to recover before the start of the recording sessions.

Electrophysiological recordings were carried out in alert behaving animals placed in ventialted small $(6 \times 6 \times 6 \mathrm{~cm})$ containers. Recordings were carried out with the help of high-impedance probes $\left(2 \times 10^{12} \Omega, 10 \mathrm{pF}\right)$ connected to differential amplifiers within a bandwidth of $0.1-10 \mathrm{kHz}$ (P511, Grass-Telefactor, West Warwick, RI, USA). Electrical stimulations were provided across isolation units connected to a CS-220 stimulator (Ciberted, Madrid, Spain).

For input/output curves, monosynaptic fEPSPs were evoked in the CA1 area by single (100 $\mu$ s, square, and negative-positive) pulses applied to Schaffer collaterals. These pulses were presented at increasing intensities ranging from $20 \mu \mathrm{A}$ to $400 \mu \mathrm{A}$, in steps of $20 \mu \mathrm{A}$. In order to avoid interactions with the preceding stimuli, an interval of $30 \mathrm{~s}$ was allowed between each pair of pulses (Madronal et al., 2007).

For the paired-pulse facilitation at the CA3-CA1 synapse, we used the same type of pulses indicated above, but presented in pairs at increasing inter-pulse intervals $(10,20,40,100,200$ and $500 \mathrm{~ms})$. For each animal, the stimulus intensity was set at $30-40 \%$ of the intensity necessary for evoking a maximum fEPSP response (Gruart et al., 2006; Gureviciene et al., 2004). An additional criterion for selecting stimulus intensity was that a second stimulus, presented $40 \mathrm{~ms}$ after a conditioning pulse, evoked a larger (20\%) synaptic field potential (Bliss and Gardner-Medwin, 1973). Intervals between pairs of pulses were set at $30 \mathrm{~s}$, to avoid unwanted interactions evoked by pre- or post-synaptic mechanisms. 
a
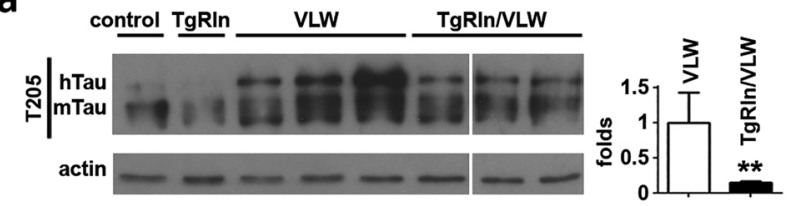

b

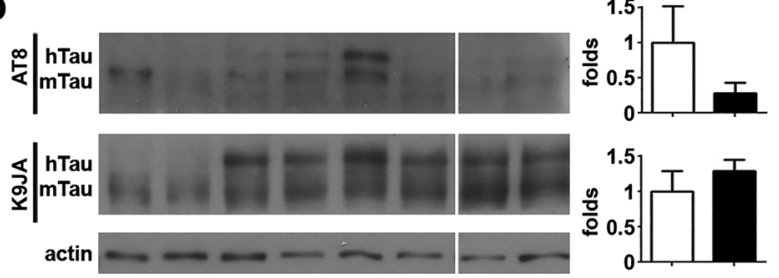

Fig. 1. Reelin reduces Tau phosphorylation in VLW model of Tauopathy. Hippocampal protein extracts from 6- month-old control, TgRln, VLW and TgRln/VLW mice were subjected to WB analysis of AD-related phospho-Tau epitopes T205 (a) and AT8 (b). Reelin overexpression in TgRln/VLW mice modulates the elevated phosphorylation of the human Tau isoform found in VLW mice. Changes in phospho-Tau are not due to variations in total Tau (K9JA) (b). $\mathrm{n}=3-4$ animals per genotype. Data are represented as mean $\pm \mathrm{SEM} ;{ }^{* *} p<0.01$; the Student's $t$-test.

For evoking LTP, we used a high-frequency stimulation (HFS) train consisting of five $200 \mathrm{~Hz}, 100 \mathrm{~ms}$ trains of pulses at a rate of $1 / \mathrm{s}$. This protocol was presented six times, at intervals of $1 \mathrm{~min}$. As indicated above for functional synaptic plasticity, pulse intensity (50-400 A) was set at $30-40 \%$ of the amount required to evoke a maximum fEPSP response for baseline recordings $(15 \mathrm{~min})$ and after the HFS train (60 min). Recording sessions were repeated for 4 additional days (30 min each) To avoid evoking a population spike and/or unwanted electroencephalographic (EEG) seizures, the stimulus intensity during the HFS train was set at the same amount as that used for generating baseline recordings.

\subsection{Statistical analysis}

Statistical analysis was performed using GraphPad Prism software. Outlier values were identified by applying the ESD method (extreme studentized deviate) and excluded from the analysis.

For quantification of WB in Fig. 1 ( $n=3-4$ animals per genotype), densitometric analysis of protein bands of interest was performed using GelPro software and normalized for loading controls (i.e. actin). To include data obtained from different gels, a specific sample was loaded in each gel and used for normalization. Significance between the two genotypes expressing human Tau was analyzed by using the unpaired Student's $t$-test

To determine Tau- and NF200-missorting in neuronal cultures upon treatment we calculated the percentage of neurons with missorting in each field and normalized the values for the percentage of missorting in untreated neurons. Significance between groups was analyzed using the one-way ANOVA with Newman-Keuls post hoc test; $\mathrm{n}=2-3$ replicates per condition from 3 to 4 independent experiments.

To determine the intensity of Tau and NF200 staining in primary neuronal cultures, five individual measures were performed per field both for translocated and untranslocated dendrites in a double-blind quantification of images. Significance between groups was analyzed at a distance of $5 \mu \mathrm{m}$ from cell soma using the one-way ANOVA with Newman-Keuls post hoc test; $\mathrm{n}=2-3$ scannings per condition from 3 independent experiments.

T205-positive signal in granule cell somas and pyramidal dendrites in CA1 were determined in sections from 1.35 to $2.30 \mathrm{~mm}$ posterior to Bregma. Significance between groups was analyzed for each area using the one-way ANOVA with Newman-Keuls post hoc test; $n=3-5$ animals per genotype. PHF-1-positive mossy fibers in the stratum lucidum was determined in sections from 1.35 to $2.30 \mathrm{~mm}$ posterior to Bregma. Significance between groups was analyzed using the one-way ANOVA with Newman-Keuls post hoc test; $n=3-4$ animals per genotype. To determine the density of PHF-1-positive cells in the hippocampal dentate gyrus (from $1.35 \mathrm{~mm}$ to $2.30 \mathrm{~mm}$ posterior to Bregma), we counted PHF-1-stained cell somas in the granular layer (GL); data were normalized to the longitude of the GL layer counted in $30-\mu \mathrm{m}$-thick sections containing one hemisphere. Longitudes measured for quantification were determined using ImageJ on images obtained with NDPview2 from Nanozoomer scans of the preparations. Significance between groups was analyzed using the one-way ANOVA with Newman-Keuls post hoc test; $n=3-4$ animals per genotype.

To determine significant differences between groups in the inputoutput test, we quantified the slopes of fEPSPs evoked at CA3CA1 synapses. fEPSPs were evoked with increasing intensities (from 0.02 to $0.4 \mathrm{~mA}$ in steps of $0.02 \mathrm{~mA} ; 10$ times for each intensity). Data evolution and differences between groups were determined using the two-way repeated measures ANOVA followed by the Holm-Sidak method ( $\mathrm{n} \geq 9$ animals per genotype). The double-pulse facilitation test was carried out at the same synapse ( $n \geq 8$ animals per genotype) in the four groups at six different intervals (10 times for each interval). Again, differences between groups were determined using the two-way repeated measures ANOVA followed by the Holm-Sidak method. Finally, LTP was evoked in the four groups ( $\mathrm{n}=10$ animals per genotype) for five recording sessions following baseline recordings and the high-frequency stimulation (HFS) train above described. fEPSPs were evoked at the CA3-CA1 synapse every $20 \mathrm{~s}$ and averaged each $5 \mathrm{~min}$ ( $n=15$ recordings per average) for analysis and representation. Significant differences with baseline recordings and between groups were determined using the two-way repeated measures ANOVA followed by the Holm-Sidak method.

To quantify behavioral performance, distances travelled during the Open field test were represented and analyzed separately for the $\mathrm{X}, \mathrm{Y}$ and $\mathrm{Z}$ axes. Time spent in the central and periphery areas of the cage were also evaluated. Differences between groups were determined using the one-way ANOVA with Newman-Keuls post hoc test. $\mathrm{n}=8-15$ animals per genotype. Latencies in the Elevated path were represented and analyzed using the two-way ANOVA (repeated measures in Test 1 and Test 2 for the same animals) followed by Bonferroni post-test; $\mathrm{n}=10-14$ animals per genotype. Additionally, a Number of fallen and latency to first fall in the Rotarod test were represented and analyzed to determine differences between groups using the one-way ANOVA with Newman-Keuls post hoc test; $\mathrm{n}=10-19$ animals per genotype. Passive Avoidance data were represented as cumulative curves in both times points of $0 \mathrm{~h}$ (habituation) and $24 \mathrm{~h}$ and analyzed separately. Significance between genotypes was determined by curve comparisons in pairs using Log-Rank (Mantel Cox) Test for each time point of analysis; $\mathrm{n}=10-19$ animals per genotype.

\section{Results}

\subsection{Reelin and Tau phosphorylation in mouse models of Tauopathy}

To explore the effect of Reelin on Tau phosphorylation in Tauopathy mouse models, we first crossbred Reelin-overexpressing mice (TgRln) (Pujadas et al., 2010; Pujadas et al., 2014) with the Tauopathy mouse model VLW (overexpressing a 4-repeat isoform of human Tau bearing three Fronto temporal dementia linked with parkinsonism-17 mutations (G272 V, P301 L and R406W) (Lim et al., 2001)).

Transgenic mice overexpressing both Reelin and mutated Tau (VLW), referred to as TgRln/VLW mice, were analyzed for Tau phosphorylation levels. In VLW mice, the Tau transgene is directed by insertion of the cDNA into a murine thy 1 gene expression cassette and is thus constitutively expressed in pyramidal neurons distributed across the cortex and hippocampus, prominently in pyramidal neurons of the CA1 and in granular cells of the DG, partially overlapping with Reelin 
transgene expression (Suppl. Fig. 1). We performed WB analysis on hippocampal extracts from TgRln, VLW, and double TgRln/VLW transgenic mice, as well as on control littermates. Regarding the transgenic isoform of human Tau (higher molecular weight band), VLW mice showed a prominent signal of the T205 phosphorylated epitope (Davila-Bouziguet et al., 2019), while Reelin overexpression led to an $85 \%$ reduction in T205 signal (Fig. 1a). Hyperphosphorylation of another Tau epitope was also tested using the antibody AT8, which recognizes phosphorylation on serines 199-202/threonine 205 and has been associated with early phases of Tauopathy (Wang and Mandelkow, 2016; Bertrand et al., 2010). The AT8 epitope in VLW transgenic animals showed a non-significant $60 \%$ decrease when Reelin was cooverexpressed (Fig. 1b). Finally, total levels of Tau were not significantly altered in any genotype by Reelin overexpression, as determined by WB using the phosphorylation independent antibody K9JA (Fig. 1b).

We also crossbred the TgRln strain with another Tauopathy model, namely GSK-3 $\beta$ AD mice (Lucas et al., 2001), providing simultaneous overexpression of Reelin and the Tau kinase GSK-3 $\beta$ in the striatum, cerebral cortex and hippocampus (Suppl. Fig. 1). In agreement with previous studies (Wang and Mandelkow, 2016; Lim et al., 2001; Bertrand et al., 2010; Engel et al., 2006), our data suggest an increase in Tau phosphorylation in GSK- $3 \beta$ mice that is not observed in TgRln/
GSK-3 $\beta$ mice (Suppl. Fig. 2).

Taken together, our data suggest that Reelin overexpression may cause a reduction in the extent of Tau phosphorylation in in vivo Tauopathy models. Given that this was not caused by altered levels of total Tau, our data suggest a Reelin-dependent reduction of phosphoTau levels.

\subsection{Reelin reduces A $\beta$-induced mislocalization of Tau and NF into the somatodendritic compartment}

In addition to altered Tau phosphorylation levels, one of the early events of Tauopathy is the missorting of endogenous Tau from the axon to the somatodendritic compartment. This missorting is accompanied by the redistribution of other axonal cytoskeletal proteins, such as NF, which also undergoes a loss of polarized distribution. In the context of $\mathrm{AD}$ modeling, mislocalization of axonal proteins can be induced by amyloid species (Zempel et al., 2013; Zempel et al., 2010). To address the effect of Reelin on the missorting of axonal cytoskeletal proteins, we treated mouse primary hippocampal neurons (21 DIV) for $3 \mathrm{~h}$ with either oligomeric species of $A \beta_{42}(1-3 \mu \mathrm{M})$ in the form of $A \beta$-derived diffusible ligands (ADDLs) (Lambert et al., 2001) or with vehicle, plus either purified Reelin or Mock-control. We then examined the subcellular localization of Tau and NF in response to the treatments. The
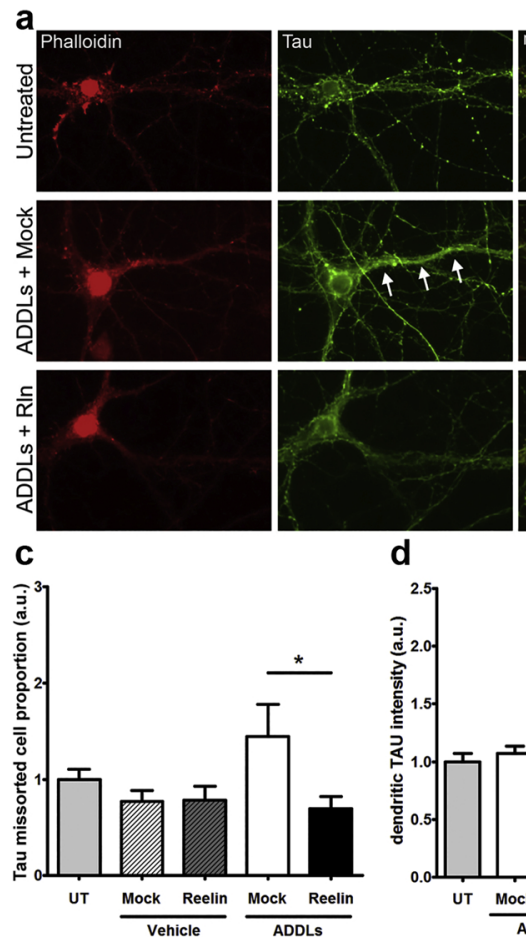
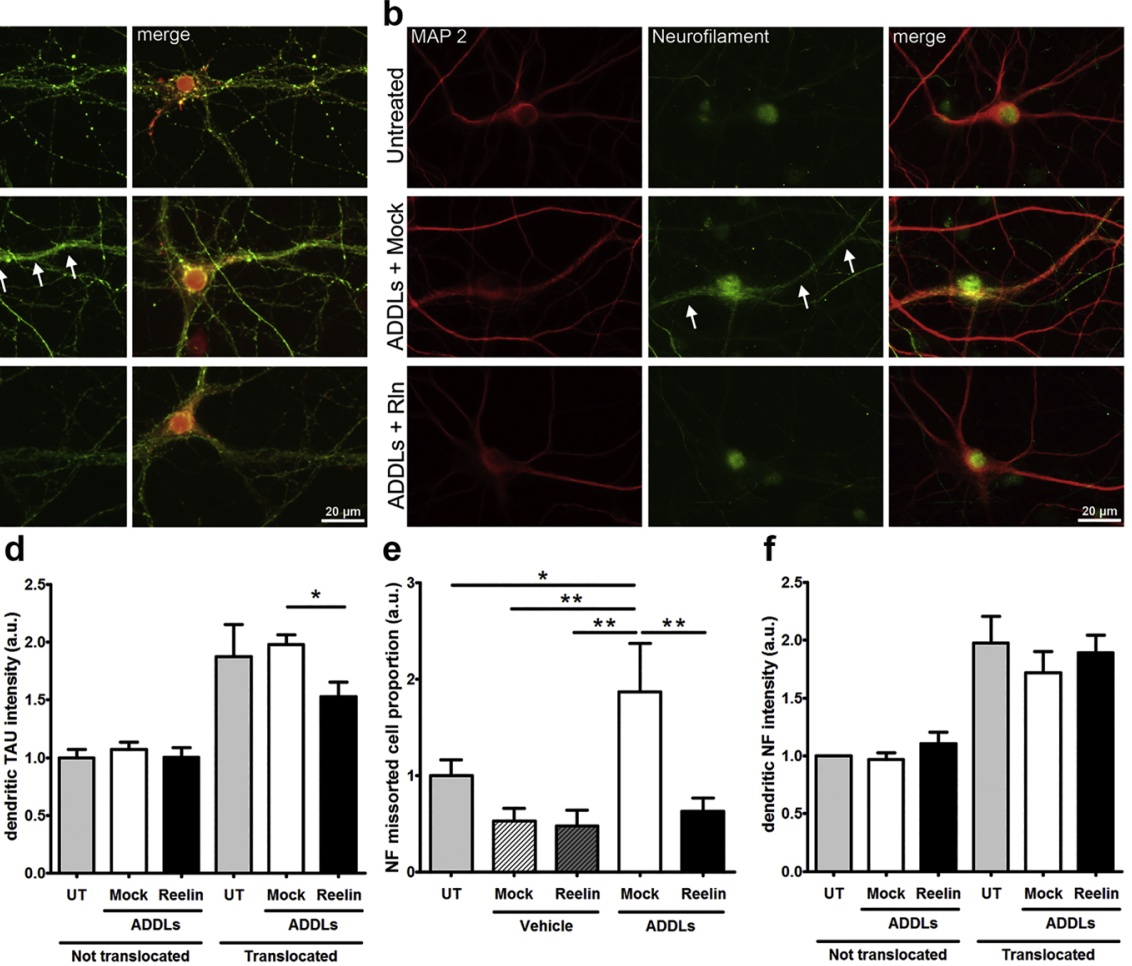

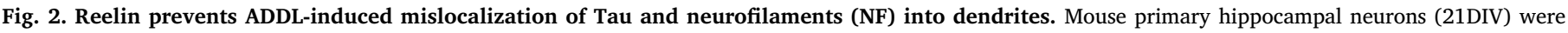

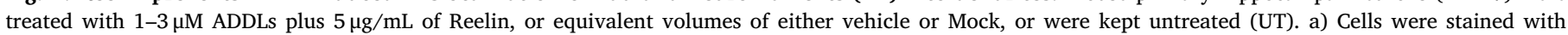

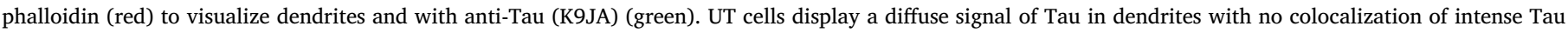

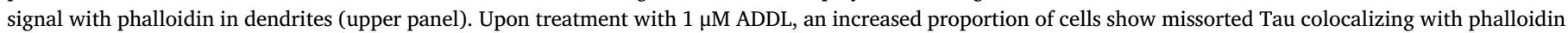

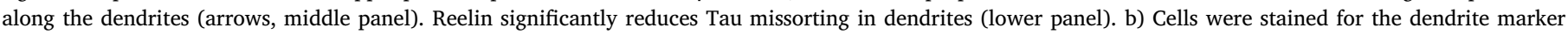

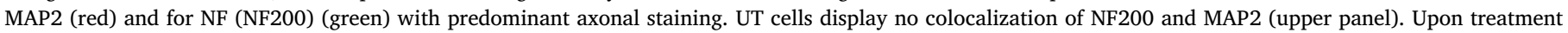

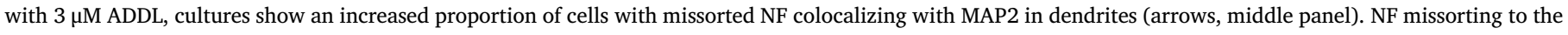

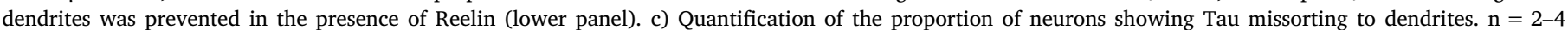

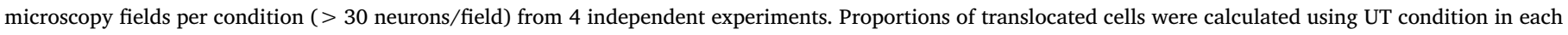

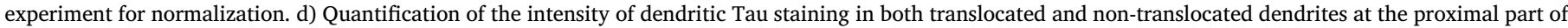

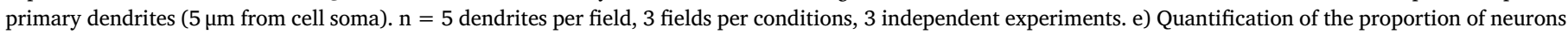

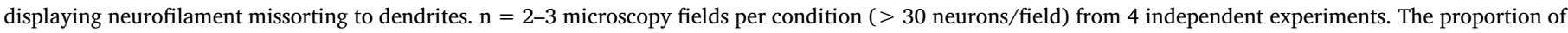

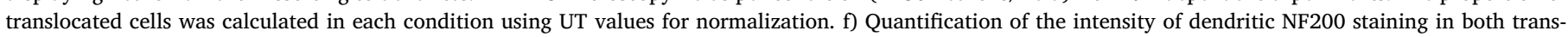

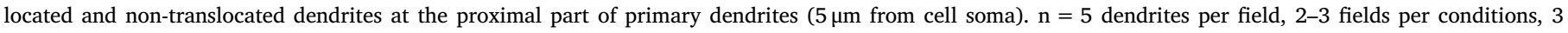
independent experiments. Data are presented as mean \pm SEM; ${ }^{*} p<0.05 ;{ }^{*} p<0.01 ; * * * p<0.001$; one-way ANOVA, Newman-Keuls post hoc test. 
proportion of Tau and NF missorting was determined by counting the neurons displaying at least one Tau/NF-translocated dendrite.

Exposure of the cultures to co-treatments of ADDLS $(1 \mu \mathrm{m})$ and Reelin $(5 \mu \mathrm{g} / \mathrm{mL})$ led to a significant decrease in the proportion of neurons with missorted Tau compared to treatment with ADDLs alone, thereby indicating that the presence of Reelin caused a reduction in the missorting phenotype (Fig. 2a,c). A stronger phenotype was observed with NF missorting upon treatment with ADDLs. Exposure of the cultures to up to $3 \mu \mathrm{M}$ ADDLs induced a significant increase in NF missorting, as compared with vehicle-treated or untreated neurons. Cotreatment with Reelin $(5 \mu \mathrm{g} / \mathrm{mL})$ completely reversed the proportion of neurons exhibiting missorted NF (Fig. 2b,e).

To further analyze the effect of Reelin on the distribution of axonal proteins, the intensity of fluorescence signal was quantified in translocated dendrites both for NF and Tau proteins. In untreated and mock neurons, the intensity of the Tau signal in translocated dendrites was 1.5- to 2-fold higher than in neurons with non-translocated Tau (Fig. 2d,f). The increased intensity of Tau in translocated neurons was significantly reduced in neurons treated with Reelin/ADDLs, as compared to those treated with ADDLs alone (Fig. 2f). NF intensity along translocated dendrites was similar in all the treatment conditions, thereby indicating that ADDLs and Reelin modulate the number of neurons displaying translocated NF but do not alter the extent of this translocation (Fig. 2f).

These results indicate that Reelin rescues in vitro the $A \beta$-induced somatodendritic missorting of axonal cytoskeletal proteins such as Tau and NF. Moreover, Reelin is able to modulate the fine distribution of Tau protein inside the translocated dendrites, resulting in the reduced accumulation of this protein in the proximal part of the dendrites.

\subsection{Reelin reverts in vivo the somatodendritic localization of phosphorylated Tau in the hippocampus of the VLW mouse model of Tauopathy}

To analyze the in vivo effect of Reelin on the redistribution of phospho-Tau, we performed immunohistochemistry with phospho-Tauspecific antibodies in hippocampal sections from TgRln/VLW mice, as well as from control, TgRln and VLW littermates.

We first studied the in vivo distribution of phospho-Tau in the VLW Tauopathy model and performed immunohistochemistry using the T205 antibody. Animals from all the genotypes displayed immunoreactivity in interneurons distributed throughout all hippocampal layers. In addition, VLW mice showed an overall increase in phosphorylation in the neuropil, and intense somatodendritic staining of CA1-CA3 pyramidal cells and granule cells in the dentate gyrus, resulting in clear labeling of apical dendrites (Fig. 3a,b,c). This phenotype was not found in control mice. TgRln/VLW mice showed weaker T205 staining in apical dendrites of CA1 pyramidal cells and in granule cells (Fig. 3a,b,c). TgRln/VLW mice showed a decrease in the area of stratum radiatum occupied by T205-stained apical dendrites of CA1 (Fig. 3d, upper panel). A similar reduction was found in the area occupied by T205-positive granule cell bodies in TgRln/VLW as compared to VLW mice (Fig. 3d, lower panel).

Additionally, we also studied the distribution of phospho-Tau in the GSK-3 $\beta$ AD model using the PHF-1 antibody. GSK- $3 \beta$ animals showed a decrease in PHF-1 staining in mossy fibers compared to those of Control and TgRln mice that was not caused by the loss of mossy fibers. Interestingly, we found that $\mathrm{TgR} \ln / \mathrm{GSK}-3 \beta$ animals exhibited a reduction of the mossy fiber phenotype (Suppl. Fig. 3a-c). Concomitant with PHF-1-positive axonal loss, GSK-3 $\beta$ mice showed a significant increase in the density of somatodendritic PHF-1-positive cells in the dentate granular layer that was slightly reduced (not significant) in TgRln/GSK$3 \beta$ mice (Suppl. Fig. 3d,e).

Taken together, our in vitro and in vivo data suggests that Reelin prevents the somatodendritic missorting of phospho-Tau protein in AD mouse models.

\subsection{Reelin overexpression reverts LTP deficits in the VLW Tauopathy model}

To unravel the physiological consequences produced by alterations in Tau, we next determined the modulatory effects of Reelin on the electrophysiological properties of hippocampal circuits in the VLW Tauopathy model for the four experimental groups (control, TgRln, VLW, and TgRln/VLW). For this, we recorded input/output curves, paired-pulse facilitation, and LTP evoked at the CA3-CA1 synapses in alert behaving animals (Suppl. Fig. 4)

We analyzed the response of CA1 pyramidal neurons to single pulses of increasing intensity $(0.02-0.4 \mathrm{~mA})$ presented to the ipsilateral CA3 area. As illustrated in Fig. 4a, the four groups of mice presented similar increases in fEPSP slopes suggesting a normal basal functioning of CA3CA1 synapses in all the genotypes analyzed (Madronal et al., 2009) $\left[F_{(57,703)}=0.518 ; p=0.999\right]$. It is generally accepted that changes in synaptic strength evoked by a pair of pulses are a form of presynaptic short-term plasticity, mostly related to variations in neurotransmitter release (Zucker and Regehr, 2002). In particular, paired-pulse stimulation is experimentally used as an indirect measurement of changes in the probability of neurotransmitter release at presynaptic terminals of hippocampal synapses (Zucker and Regehr, 2002) even in behaving mice (Madronal et al., 2009). Paired-pulse facilitation evoked in the four groups of mice was analyzed presenting a fix stimulus intensity (30-40\% of asymptotic values) with increasing inter-pulse intervals (Fig. 4b). The four groups of mice presented paired pulse facilitations at short $(20$ and $40 \mathrm{~ms})$ inter-pulse intervals $\left[\mathrm{F}_{(5,299)}=19.635\right.$; $\mathrm{p}<0.001]$. However, no significant differences were observed between groups $\left[\mathrm{F}_{(15,230)}=0.551 ; \mathrm{p}=0.91\right]$.

In a final experimental step, we carried out an LTP study in the four groups of mice. As known, CA3-CA1 synapses are involved in the acquisition of different types of associative and non-associative learning tasks and it is usually selected for evoking LTP in behaving mice (Gruart et al., 2006; Madronal et al., 2007; Madronal et al., 2009). For baseline values, animals were stimulated in the CA3 area at a rate of 3 times/ min for $15 \mathrm{~min}$ (Fig. 4c). Animals were then presented with a HFS protocol (see Methods). Following the HFS train, the same single stimulus used to generate baseline records was presented at the initial rate (3/min) for another $60 \mathrm{~min}$. Recording sessions were repeated for four additional days (30 min each; Fig. 4c). While control and TgRln mice presented a significant $\left[\mathrm{F}_{(114,1368)}=5.328 ; \mathrm{p}<0.001\right]$ increase in fEPSP slopes following the HFS session, the VLW mice failed to present a significant LTP ( $p \geq 0.998$ ). This failure was not seen with Reelin overexpression in TgRln/VLW mice leading to a complex scenario when VLW Tau mutation is counteracted by Reelin.

In addition, a point to point comparison between fEPSPs evoked in the four groups of mice after the HFS protocol indicated that the control, TgRln, and TgRln/VLW groups presented significantly larger and longer-lasting ( $\mathrm{p} \leq 0.05$ ) increases in fEPSP values than the VLW group. Interestingly enough, the TgRln group presented significantly larger and longer-lasting increases in fEPSP values than control and TgRln/ VLW groups (Fig. 4c).

In conclusion, the four groups of mice presented similar basal synaptic properties and short-term plasticity. In contrast, VLW mice only presented a modest and non-significant LTP and significantly lower fEPSP increases in fEPSP values. These LTP deficits were not further observed following overexpression of Reelin in TgRln/VLW mice.

\subsection{Reelin overexpression reverts cognitive impairment in the VLW Tauopathy model}

To evaluate the physiological impact of the Reelin-induced modulations on the phosphorylation and distribution of Tau, animals from the different genotypes (control, TgRln, VLW and TgRln/VLW) were subjected to behavioral studies. We first examined motor performance, observing no relevant differences between genotypes for the open field (Fig. 5a), the rotarod test (Fig. 5b) or the elevated path (Fig. 5c). The 


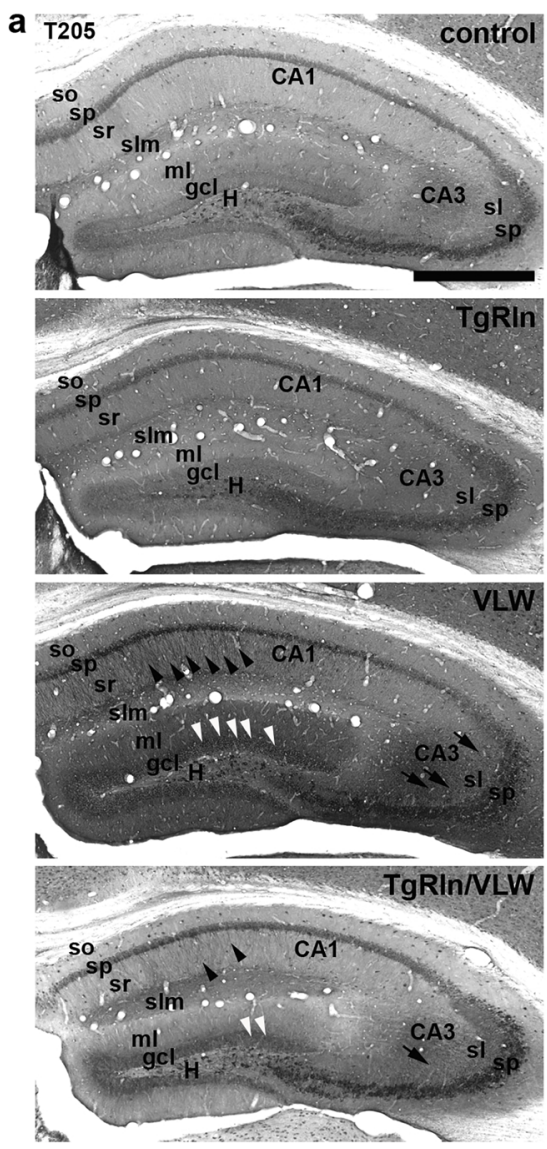

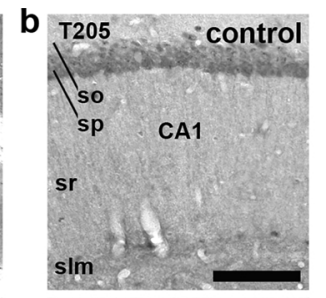
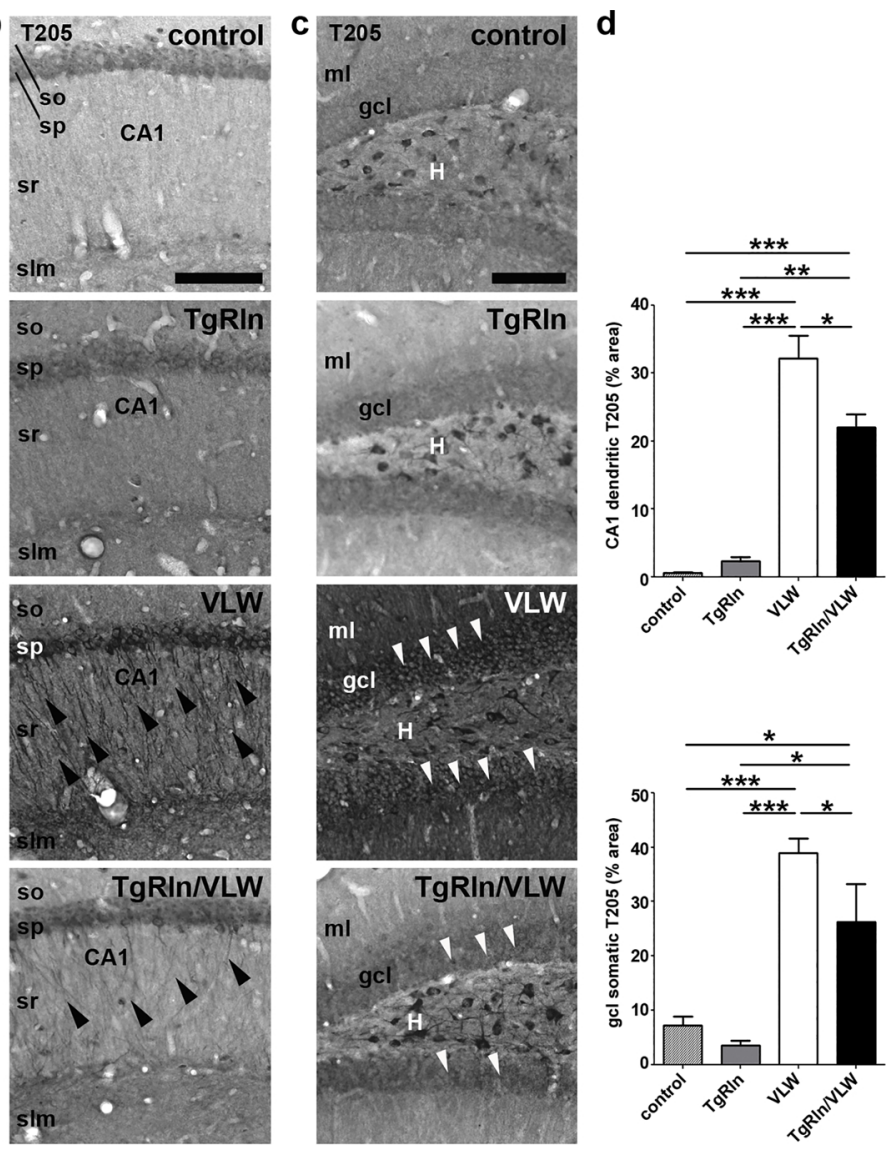

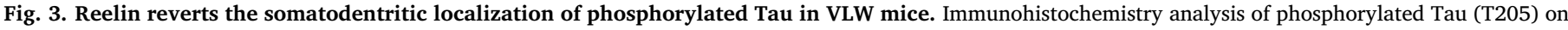

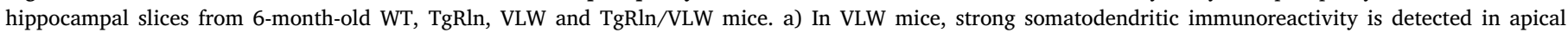

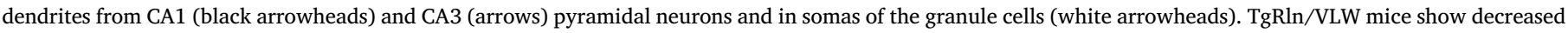

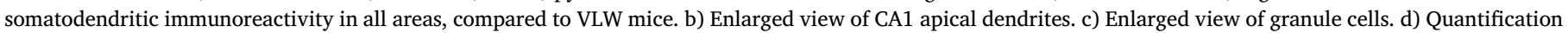

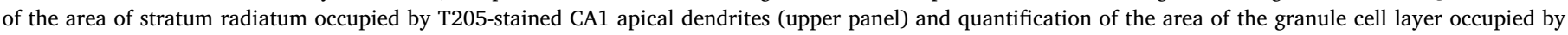

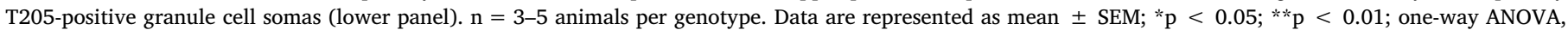

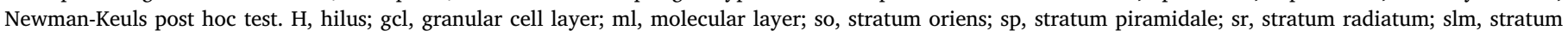
lacunosum moleculare; sl, stratum lucidum.

cognitive performance of the animals was also evaluated in the fearmotivated test of passive avoidance. During the acquisition phase of the test, animals of the different genotypes showed identical latencies to enter to the dark area, with an average time below $20 \mathrm{~s}$ (Fig. $5 \mathrm{~d}$ and Suppl. Table 1). After $24 \mathrm{~h}$, animals were subjected to the same paradigm to assess long-term memory. Control mice and TgRln littermates had an average latency of over $100 \mathrm{~s}$ to enter the dark area, thereby indicating memory retention. In contrast, VLW mice showed lower latencies, thus pointing to impaired memory caused by their Tauopathy phenotype. Finally, the performance of TgRln/VLW mice, which show co-expression of Reelin and mutated Tau, was indistinguishable from that of control mice. This observation thus points to full reversal of the cognitive alteration in this paradigm upon Reelin overexpression (Fig. 5d and Suppl. Table 1). These results indicate that VLW mice did not present evident motor deficits; however, they did show significant deficits in memory retention. In addition, these findings point to the physiological relevance of the Reelin-induced reduction in phosphorylation levels and aberrant distribution of Tau in VLW mice. Finally, our results indicate that Reelin overexpression can counteract Tauopathyrelated histological, electrophysiological and behavioral abnormalities.

\section{Discussion}

Tau protein is a major player in neurodegeneration and can be considered a convergent therapeutic target for a diversity of neurodegenerative diseases. Indeed, alterations in the phosphorylation, structural conformation and subcellular distribution of Tau are crucial for the pathogenesis of the most frequent dementias, including $\mathrm{AD}$, frontotemporal dementias with parkinsonism linked to chromosome 17 (FTDP-17), progressive supranuclear palsy (PSP), corticobasal degeneration (CBD), and Pick's disease (Wang and Mandelkow, 2016; Buee and Delacourte, 1999; Gao et al., 2018; Falcon et al., 2018). Regarding $\mathrm{AD}$, recent evidence has highlighted a major role of Tau in the pathogenesis of the disease with links to neuroinflammation and prion-like mechanisms (Wang and Mandelkow, 2016; Gao et al., 2018; Goedert et al., 2017; Hickman et al., 2018; Soto and Pritzkow, 2018). Importantly, Tau is a mediator of $\mathrm{A} \beta$ toxicity, as demonstrated in in vitro and in vivo experiments showing that amyloidosis per se is not pathological in animal models with a Tau knock-out background (Roberson et al., 2007; Roberson et al., 2011; Chabrier et al., 2012; Chabrier et al., 2014). In vitro, $\mathrm{A} \beta$ requires Tau protein for toxicity and promotes Tau alterations by altering its phosphorylation state and its fine subcellular distribution (Li and Gotz, 2017). Moreover, in pathological conditions, the phosphorylation of Tau at AD-related epitopes (e.g. PHF-1 and AT8) is believed to start in the axon, resulting in the detachment of Tau from microtubules and in its redistribution to the somatodendritic compartment (Bertrand et al., 2010; Sohn et al., 2016). This process finally triggers the destabilization of axons and consequent degeneration (Kneynsberg et al., 2017). In parallel, the mislocalization of Tau allows it to mediate $\mathrm{A} \beta$-induced toxicity in the dendritic compartment, thereby 
a

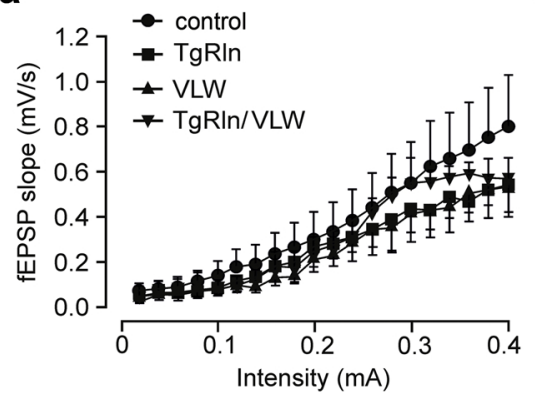

b

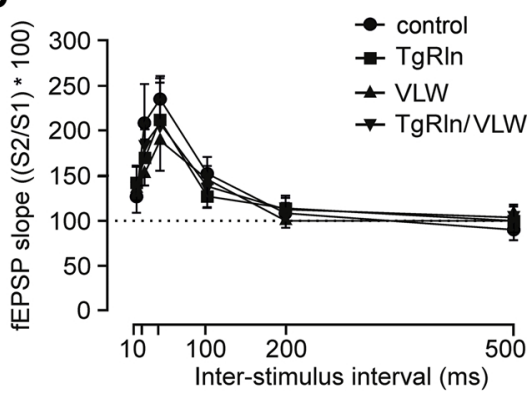

C
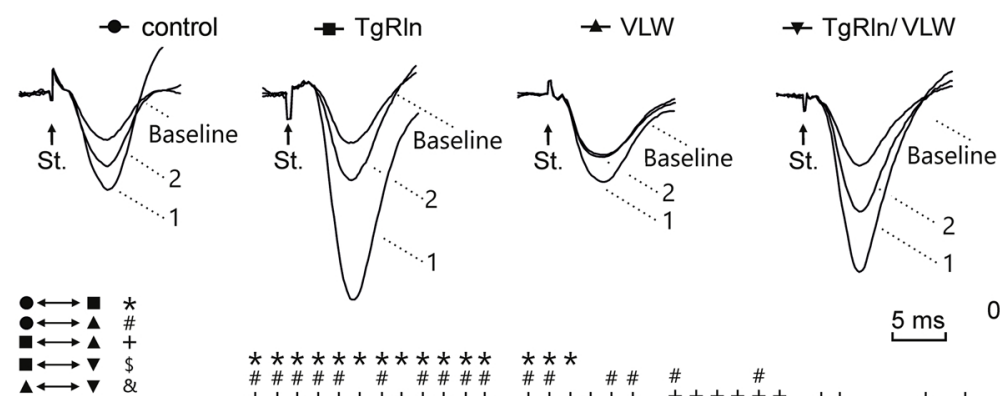

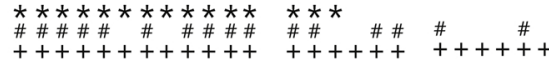

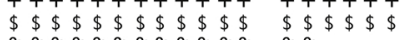

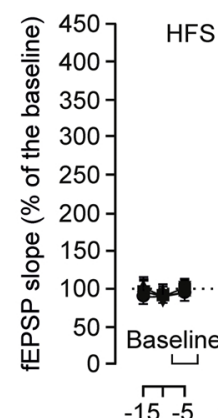

HFS:

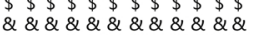

$\& \&$

\& \& \& \& \& \& \&
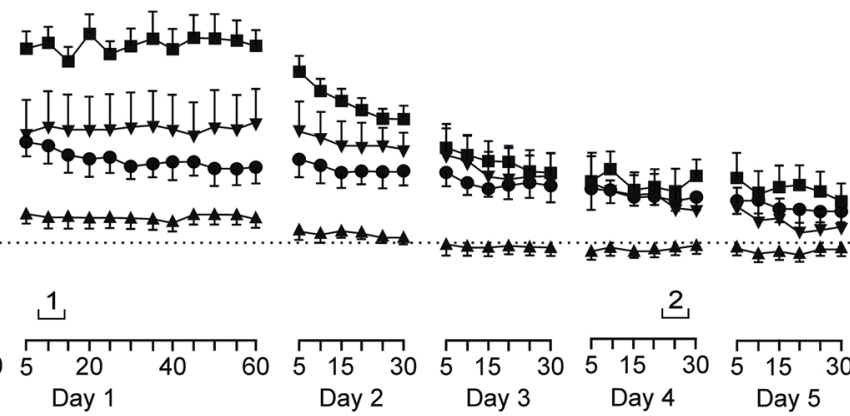

Day 2

Time (minutes)
Fig. 4. Reelin overexpression increases LTP in control animals and reverts LTP deficits in the VLW model. a) Input/output curves of field excitatory post-synaptic potentials (fEPSPs) evoked at the CA3-CA1 synapse by single pulses of increasing intensities (0.02-0.4 in $\mathrm{mA}$ ) in control (black circles), TgRln (black squares), VLW (up black triangles), and TgRln/VLW (down black triangles) ( $\mathrm{n} \geq 9$ animals per group). Data is represented as mean \pm SEM. Note the progressive in fEPSP slopes with the increase in stimulus intensities. No significant differences ( $\mathrm{p}=0.727$; two-way repeated measures ANOVA) were observed between the four groups. b) Double pulse facilitation evoked in the four experimental groups ( $\mathrm{n} \geq$ 8 animals per group). Data shown are mean \pm SEM slopes of the 2nd fEPSP expressed as the percentage of the 1 st for six $(10,20,40,100,200,500)$ inter-pulse intervals. The four groups presented a facilitation at short $(20-40 \mathrm{~ms})$ inter-pulse intervals, with no significant differences ( $p=0.766$; two-way repeated measures ANOVA) between them. c) Graphs illustrating the time course of LTP evoked in the CA3-CA1 synapse following an HFS session presented to mice included in the four experimental groups ( $n=10$ animals per group). After $15 \mathrm{~min}$ of baseline recordings, animals were presented with the high-frequency stimulation (HFS) train described in Methods and indicated by the dashed line. LTP evolution was followed for five days. At the top are illustrated representative examples of fEPSPs collected at the times indicated in the bottom graphs collected from a representative animal of each group. fEPSP slopes are given as a percentage of fEPSP values collected during baseline recordings (100\%). Three (control, TgRln, and TgRln/VLW) of the groups presented significant ( $\mathrm{p} \leq 0.05$ ) increases in fEPSP values following the HFS, but not the VLW one ( $p \geq 0.998$ ). These three groups presented significantly $(p \leq 0.05)$ larger fEPSP values than the VLW group. In addition, the group overexpressing Reelin (TgRln) also presented significantly ( $\mathrm{p} \leq 0.05$ ) larger values than control and TgRln/VLW groups (two-way repeated measures ANOVA). causing excitotoxic synaptic alterations (Ittner and Gotz, 2011; Ittner et al., 2010). Indeed, phospho-Tau accumulation in the synaptic compartment contributes to synaptic dysfunction (Baglietto-Vargas et al., 2018).

To test the potential of the extracellular protein Reelin as a putative therapeutic tool for Tauopathy-associated diseases, here we used various in vivo and in vitro assays of Tau-related pathogenesis (phosphorylation, mislocalization, and cognitive impairment), in a variety of conditions linked or not to $A \beta$ toxicity. Our results indicates that, in addition to delaying $A \beta$ deposition in vivo and rescuing synaptic loss and cognitive impairment in amyloid mouse models (Pujadas et al., 2014), the overexpression of Reelin counteracts at least two pathological hallmarks of Tau, namely the somatodendritic missorting of Tau induced by $A \beta$ and the cognitive impairment associated with pathological Tau mutations in the VLW model. In addition, the present findings show a trend for Reelin to reduce Tau phosphorylation. Thus, on the basis of previous studies (Kocherhans et al., 2010; Doehner et al., 2010; Knuesel et al., 2009; Krstic et al., 2013) and our own findings, Reelin emerges as a possible, convergent therapeutic target in AD-related diseases by ameliorating both $\mathrm{A} \beta$ - and Tau-associated pathologies.
4.1. Reversal of somatodendritic Tau and NF mislocalization by Reelin in both $A \beta$-dependent and -independent manners

Our data show that Reelin reverses A $\beta$-induced NF- and Tau-translocation. Indeed, Reelin treatment decreased the proportion of neurons displaying somatodendritic localization of NF and Tau proteins upon treatment with ADDLs. However, slight differences were found in the degree of rescue for each protein. The signal intensity in proximal dendrites was modulated by Reelin in the case of Tau, but not in the case of NF protein, which may be related to the different concentrations used: Tau mislocalization was already induced by incubation with $1 \mu \mathrm{M}$ ADDLs, whereas $3 \mu \mathrm{M}$ treatments were needed to stimulate NF translocation. These observations thus suggest that Tau protein shows greater sensitivity to $A \beta$-induced mislocalization. It has been shown that missorted Tau originates from newly synthesized protein in the cell body (Zempel et al., 2013) and that the aberrant distribution of Tau is likely to be caused by selective alterations in the axon initial segment, a key structure in the targeting of neuronal proteins to the axonal and somatodendritic compartments (Zempel et al., 2017). As Reelin negatively regulates Tau phosphorylation, our results do not allow us to determine whether the blockade of Tau missorting by Reelin is due to a direct regulation of the protein targeting mechanisms or, alternatively, whether it is secondary to the decreased phosphorylation of Tau. Thus, further studies should address whether decreased Tau phosphorylation 
a

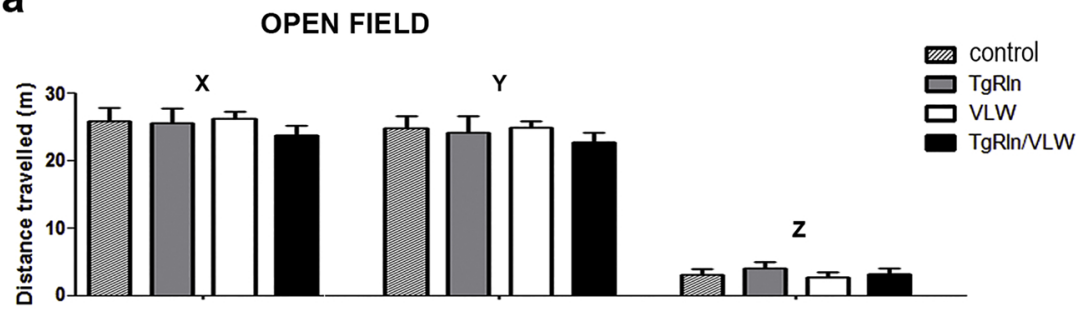

b
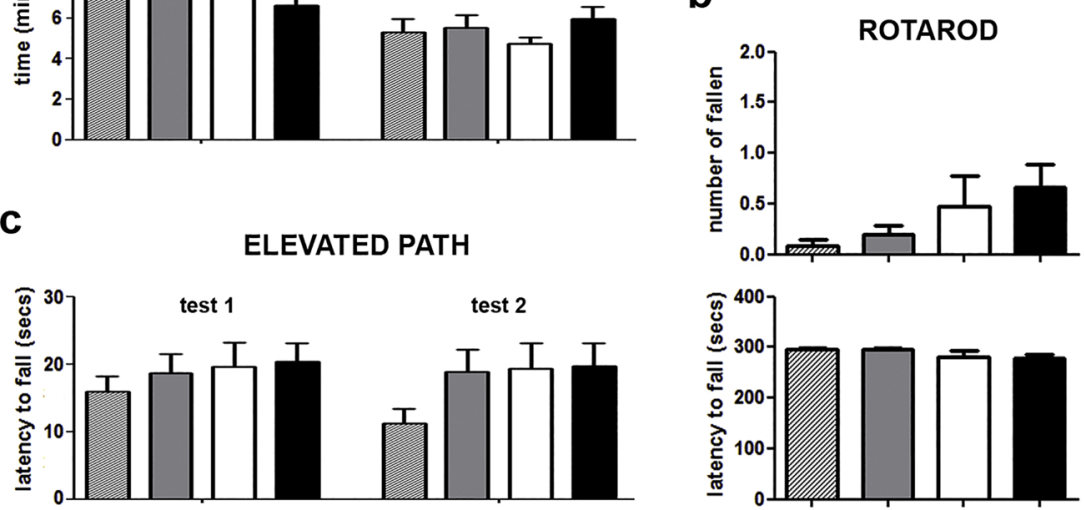

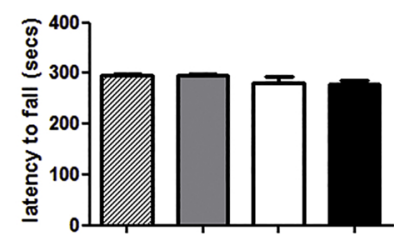

d

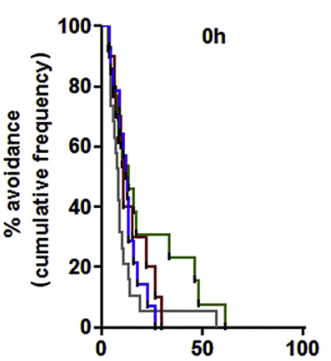

PASSIVE AVOIDANCE

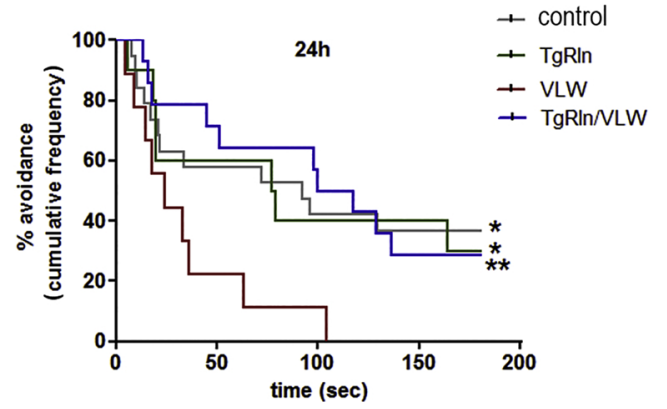

Fig. 5. Reelin overexpression rescues cognitive deficits in VLW model. a) Open field. Quantification of the mean distance travelled by mice (in $\mathrm{m}$ ) in either $\mathrm{X}, \mathrm{Y}$ and $\mathrm{Z}$ axes inside the test cage (upper panel). The time (in $\mathrm{min}$ ) that animals spent in the central area and in the periphery are also represented (lower panel). Data are represented as mean \pm SEM; no significant differences were observed between groups (one-way ANOVA). $\mathrm{n}=9-19$ animals per genotype. b) Accelerating rotarod. Bars show the mean number of falls (left) and the time (in s; right) that the animals remained on the rotating bar over the two successive trials. Data are represented as mean \pm SEM; no significant differences were observed between the four experimental groups (one-way ANOVA). $n=10-19$ animals per genotype. c) Elevated path test. The bars indicated the mean latency (in s) to fall of the four experimental groups during the two trials of the test. Data are represented as mean \pm SEM; no significant differences were observed between groups (two-way ANOVA). $n=10-14$ animals per genotype. d) Passive avoidance test. Latency to enter the dark compartment for the control, TgRln, VLW, and TgRln/VLW groups was measured to a maximum of $180 \mathrm{~s}$. Data are represented in cumulative frequencies; significant differences between genotypes was determined by curve comparisons in pairs using Log-Rank (Mantel Cox) Test for each time point of analysis s ${ }^{*} \mathrm{p}<0.05$; ** $\mathrm{p}<0.01 . \mathrm{n}=9-19$ animals per genotype. Although the latency between the four groups did not differ during the acquisition session, the latency of the VLW group during the retention session was smaller than for the other three groups. Indeed, the cumulative frequency presented by the VLW group for the retention test $(24 \mathrm{~h})$ was significantly smaller than for the other three groups. directly drives or influences the mislocalization of this protein. Additionally, the described interaction of Reelin with ADDLs (Pujadas et al., 2014) makes it necessary to analyze separately the role of Reelin in counteracting ADDLs by sequestration and the role of Reelin in directly modulating Tau localization.

We also explored the mislocalization of Tau in two in vivo models of Tauopathy in which protein mistargeting is not associated with $A \beta$ pathology. In the VLW model, the sole expression of human Tau with epitopes prone to phosphorylation promoted the aberrant distribution of phospho-Tau to the somatodendritic compartment of the neurons overexpressing this isoform, i.e., hippocampal pyramidal and granule cells. The recovered immunohistological distribution of phospho-Tau in $\mathrm{TgRln} / \mathrm{VLW}$ mice may be attributable to a reduction in total phosphoTau levels as compared to VLW animals. In the GSK-3 $\beta$ mouse model, the overexpression of this Tau kinase also promoted aberrant phosphoTau distribution in the cell bodies of the granular cells of the dentate gyrus, as described (Lucas et al., 2001). Moreover, here we also show that the mossy fibers show a reduction of phospho-Tau staining that is rescued by Reelin overexpression. Taken together, the present in vivo findings suggest that aberrant phospho-Tau mislocalization is normalized by Reelin.

Our findings in VLW mice also indicate that the overexpression of Reelin decreases the levels of Tau hyperphosphorylation caused by the expression of the human VLW Tau isoform. These findings were supported in the mouse model overexpressing the Tau kinase GSK-3ß. These results are consistent with previous studies showing the opposite effect, i.e. an increase in Tau phosphorylation in heterozygous reeler mice with reduced Reelin expression or in Reelin receptor knock-outs (Hiesberger et al., 1999; Kocherhans et al., 2010; Beffert et al., 2004). Taken together, the above results indicate that the Reelin pathway influences the phosphorylation state of Tau protein. Regarding the mechanisms of action of Reelin on Tau protein alterations, activation of the Reelin intracellular cascade, which leads to a decrease in the activity of the major kinase for Tau protein, namely GSK-3 $\beta$ (GonzalezBillault et al., 2005; Beffert et al., 2002), is likely to contribute to the observed phosphorylation changes in Tauopathy mouse models overexpressing Reelin. In addition, the recent finding that the APOE4 protein, which binds APOER2 receptors, exacerbates Tau pathology (Shi et al., 2017) suggests that the increase in extracellular Reelin in TgRln mice may reduce the binding of APOE proteins to the Reelin receptors.

In conclusion, our observations suggest that Reelin could reverse several hallmarks of Tauopathies, including the following: Tau mislocalization to the somatodendritic compartment caused by amyloid in vitro and also in an A $\beta$-independent manner in mouse models; and altered Tau phosphorylation in vivo. These observations indicate that the Reelin pathway may interfere with distinct and fundamental molecular mechanisms associated with Tau pathology. 


\subsection{Reelin reverses cognitive and physiological deficits in VLW mice}

Finally, the present study also explored the physiological relevance of Reelin on cognitive alterations associated with Tauopathies. Our results show that, in VLW transgenic mice, Reelin overexpression is sufficient to reverse the cognitive impairment in long-term memory indicating that the reversal of the Tau-pathogenic molecular and cellular mechanisms described above by Reelin has a direct impact on the cognitive physiological performance of mouse models of Tauopathy. Moreover, as already reported (Pujadas et al., 2010), Reelin overexpression increases dramatically LTP responses in hippocampal circuits while VLW mice fail to evoke LTP responses at hippocampal CA3CA1 synapses. The additive effect of Reelin overexpression in VLW background led TgRln/VLW mice to behave like controls, a complex scenario that could either be interpreted as a rescue of the Tauopathy phenotype in VLW mice or merely as a sum total of antagonistic effects.

\section{Conclusions}

On the basis of our findings, we propose that the activation of the Reelin pathway might provide an efficient therapeutic approach to ameliorate several pathological mechanisms commonly associated with Tauopathies, including AD. Thus, on the basis of our previous and present observations, we conclude that the extracellular protein Reelin positively targets several amyloid-related pathological processes (amyloid plaque load, oligomer assembly, synaptic loss, short- and long-term memory loss and A $\beta$-induced Tau translocation) (Pujadas et al., 2014), as well as several Tauopathy-associated processes. Therefore, the capacity of Reelin as a therapeutic agent targeting both amyloid- and Tau-associated pathological mechanisms and manifestations deserves further attention.

\section{Author contributions}

E.S and L.P conceived and designed the study, and planned and supervised the project. DR, AM and LP performed biochemical and histological experiments and analyzed data. J.A. generated GSK-3 $\beta$ and VLW transgenic mice. A.G., G.C.-M. and J.M.D.-G. were responsible for the electrophysiology and behavior experiments. D.R., L.P., E.S., J.M.D.-G., A.G. and J.A contributed to writing the manuscript.

\section{Declaration of Competing Interest}

Authors declare no conflict of interests.

\section{Acknowledgements}

This work was supported by grants from La Marató de TV3 Foundation to L.P. and J.M.D.-G. and from MINECO to E.S and L.P. (SAF2016-76340-R) and to A.G and J.M.D.-G. (BFU2017-82375-R). We thank Dr. Peter Davies for generously providing PHF-1 antibody; N. Carulla and A. Vázquez for help in producing ADDLs; M. Sánchez, J.M. González Martín, A. Lladó and L. Badia for technical assistance; and T. Yates for editorial help.

\section{Appendix A. The Peer Review Overview and Supplementary data}

The Peer Review Overview and Supplementary data associated with this article can be found in the online version, at doi:https://doi.org/ 10.1016/j.pneurobio.2019.101743.

\section{References}

Alcantara, S., Ruiz, M., D’Arcangelo, G., Ezan, F., de Lecea, L., Curran, T., et al., 1998. Regional and cellular patterns of reelin mRNA expression in the forebrain of the developing and adult mouse. J. Neurosci. 18, 7779-7799.
Baglietto-Vargas, D., Prieto, G.A., Limon, A., Forner, S., Rodriguez-Ortiz, C.J., Ikemura, K., et al., 2018. Impaired AMPA signaling and cytoskeletal alterations induce early synaptic dysfunction in a mouse model of Alzheimer's disease. Aging Cell, e12791.

Ballatore, C., Lee, V.M., Trojanowski, J.Q., 2007. Tau-mediated neurodegeneration in Alzheimer's disease and related disorders. Nat. Rev. Neurosci. 8, 663-672.

Beffert, U., Morfini, G., Bock, H.H., Reyna, H., Brady, S.T., Herz, J., 2002. Reelin-mediated signaling locally regulates protein kinase B/Akt and glycogen synthase kinase 3beta. J. Biol. Chem. 277, 49958-49964.

Beffert, U., Weeber, E.J., Morfini, G., Ko, J., Brady, S.T., Tsai, L.H., et al., 2004. Reelin and cyclin-dependent kinase 5-dependent signals cooperate in regulating neuronal migration and synaptic transmission. J. Neurosci. 24, 1897-1906.

Bertrand, J., Plouffe, V., Senechal, P., Leclerc, N., 2010. The pattern of human tau phosphorylation is the result of priming and feedback events in primary hippocampal neurons. Neuroscience 168, 323-334.

Bliss, T.V., Gardner-Medwin, A.R., 1973. Long-lasting potentiation of synaptic transmission in the dentate area of the unanaestetized rabbit following stimulation of the perforant path. J. Physiol. 232, 357-374.

Bloom, G.S., 2014. Amyloid-beta and tau: the trigger and bullet in Alzheimer disease pathogenesis. JAMA Neurol. 71, 505-508.

Buee, L., Delacourte, A., 1999. Comparative biochemistry of tau in progressive supranuclear palsy, corticobasal degeneration, FTDP-17 and Pick's disease. Brain Pathol. 9, 681-693.

Chabrier, M.A., Blurton-Jones, M., Agazaryan, A.A., Nerhus, J.L., Martinez-Coria, H., LaFerla, F.M., 2012. Soluble abeta promotes wild-type tau pathology in vivo. J. Neurosci. 32, 17345-17350.

Chabrier, M.A., Cheng, D., Castello, N.A., Green, K.N., LaFerla, F.M., 2014. Synergistic effects of amyloid-beta and wild-type human tau on dendritic spine loss in a floxed double transgenic model of Alzheimer's disease. Neurobiol. Dis. 64, 107-117.

Chin, J., Massaro, C.M., Palop, J.J., Thwin, M.T., Yu, G.Q., Bien-Ly, N., et al., 2007. Reelin depletion in the entorhinal cortex of human amyloid precursor protein transgenic mice and humans with Alzheimer's disease. J. Neurosci. 27, 2727-2733.

D'Arcangelo, G., Reeler, Curran T., 1998. New tales on an old mutant mouse. BioEssays: news and reviews in molecular. Cell. Dev. Biol. 20, 235-244.

Davila-Bouziguet, E., Targa-Fabra, G., Avila, J., Soriano, E., Pascual, M., 2019. Differential accumulation of Tau phosphorylated at residues Thr231, Ser262 and Thr205 in hippocampal interneurons and its modulation by Tau mutations (VLW) and amyloid-beta peptide. Neurobiol. Dis. 125, 232-244.

Doehner, J., Madhusudan, A., Konietzko, U., Fritschy, J.M., Knuesel, I., 2010. Co-localization of Reelin and proteolytic AbetaPP fragments in hippocampal plaques in aged wild-type mice. J. Alzheimer's Dis.: JAD 19, 1339-1357.

Durakoglugil, M.S., Chen, Y., White, C.L., Kavalali, E.T., Herz, J., 2009. Reelin signaling antagonizes beta-amyloid at the synapse. Proc. Natl. Acad. Sci. U. S. A. 106, 15938-15943.

Eleore, L., Lopez-Ramos, J.C., Yi, P.J., Delgado-Garcia, J.M., 2007. The cognitive enhancer T-588 partially compensates the motor associative learning impairments induced by scopolamine injection in mice. Behav. Neurosci. 121, 1203-1214.

Engel, T., Lucas, J.J., Gomez-Ramos, P., Moran, M.A., Avila, J., Hernandez, F., 2006. Cooexpression of FTDP-17 tau and GSK-3beta in transgenic mice induce tau polymerization and neurodegeneration. Neurobiol. Aging 27, 1258-1268.

Falcon, B., Zhang, W., Murzin, A.G., Murshudov, G., Garringer, H.J., Vidal, R., et al., 2018. Structures of filaments from Pick's disease reveal a novel tau protein fold. Nature 561, 137-140.

Gao, Y.L., Wang, N., Sun, F.R., Cao, X.P., Zhang, W., Yu, J.T., 2018. Tau in neurodegenerative disease. Ann. Transl. Med. 6, 175

Goedert, M., Eisenberg, D.S., Crowther, R.A., 2017. Propagation of tau aggregates and neurodegeneration. Annu. Rev. Neurosci. 40, 189-210.

Gonzalez-Billault, C., Del Rio, J.A., Urena, J.M., Jimenez-Mateos, E.M., Barallobre, M.J. Pascual, M., et al., 2005. A role of MAP1B in Reelin-dependent neuronal migration. Cereb. Cortex 15, 1134-1145.

Greenberg, S.G., Davies, P., Schein, J.D., Binder, L.I., 1992. Hydrofluoric acid-treated tau PHF proteins display the same biochemical properties as normal tau. J. Biol. Chem. $267,564-569$.

Groc, L., Choquet, D., Stephenson, F.A., Verrier, D., Manzoni, O.J., Chavis, P., 2007 NMDA receptor surface trafficking and synaptic subunit composition are developmentally regulated by the extracellular matrix protein Reelin. J. Neurosci. 27, 10165-10175.

Gruart, A., Munoz, M.D., Delgado-Garcia, J.M., 2006. Involvement of the CA3-CA1 synapse in the acquisition of associative learning in behaving mice. J. Neurosci. 26, 1077-1087.

Gureviciene, I., Ikonen, S., Gurevicius, K., Sarkaki, A., van Groen, T., Pussinen, R., et al., 2004. Normal induction but accelerated decay of LTP in APP + PS1 transgenic mice. Neurobiol. Dis. 15, 188-195.

Haass, C., Selkoe, D.J., 2007. Soluble protein oligomers in neurodegeneration: lessons from the Alzheimer's amyloid beta-peptide. Nat. Rev. Mol. Cell Biol. 8, 101-112.

Herring, A., Donath, A., Steiner, K.M., Widera, M.P., Hamzehian, S., Kanakis, D., et al., 2012. Reelin depletion is an early phenomenon of Alzheimer's pathology. J. Alzheimer's Dis.: JAD 30, 963-979.

Herz, J., Chen, Y., 2006. Reelin, lipoprotein receptors and synaptic plasticity. Nat. Rev. Neurosci. 7, 850-859.

Hickman, S., Izzy, S., Sen, P., Morsett, L., El Khoury, J., 2018. Microglia in neurodegeneration. Nat. Neurosci. 21, 1359-1369.

Hiesberger, T., Trommsdorff, M., Howell, B.W., Goffinet, A., Mumby, M.C., Cooper, J.A., et al., 1999. Direct binding of Reelin to VLDL receptor and ApoE receptor 2 induces tyrosine phosphorylation of disabled-1 and modulates tau phosphorylation. Neuron 24, 481-489.

Hoover, B.R., Reed, M.N., Su, J., Penrod, R.D., Kotilinek, L.A., Grant, M.K., et al., 2010. 
Tau mislocalization to dendritic spines mediates synaptic dysfunction independently of neurodegeneration. Neuron 68, 1067-1081.

Iqbal, K., Liu, F., Gong, C.X., Grundke-Iqbal, I., 2010. Tau in Alzheimer disease and related tauopathies. Curr. Alzheimer Res. 7, 656-664.

Ittner, L.M., Gotz, J., 2011. Amyloid-beta and tau-a toxic pas de deux in Alzheimer's disease. Nat. Rev. Neurosci. 12, 65-72.

Ittner, L.M., Ke, Y.D., Delerue, F., Bi, M., Gladbach, A., van Eersel, J., et al., 2010. Dendritic function of tau mediates amyloid-beta toxicity in Alzheimer's disease mouse models. Cell 142, 387-397.

Jagust, W., 2018. Imaging the evolution and pathophysiology of Alzheimer disease. Nat. Rev. Neurosci. 19, 687-700.

Kneynsberg, A., Combs, B., Christensen, K., Morfini, G., Kanaan, N.M., 2017. Axonal degeneration in tauopathies: disease relevance and underlying mechanisms. Front. Neurosci. 11, 572.

Knuesel, I., Nyffeler, M., Mormede, C., Muhia, M., Meyer, U., Pietropaolo, S., et al., 2009. Age-related accumulation of Reelin in amyloid-like deposits. Neurobiol. Aging 30, 697-716.

Kocherhans, S., Madhusudan, A., Doehner, J., Breu, K.S., Nitsch, R.M., Fritschy, J.M., et al., 2010. Reduced Reelin expression accelerates amyloid-beta plaque formation and tau pathology in transgenic Alzheimer's disease mice. J. Neurosci. 30, 9228-9240.

Kopke, E., Tung, Y.C., Shaikh, S., Alonso, A.C., Iqbal, K., Grundke-Iqbal, I., 1993. Microtubule-associated protein tau. Abnormal phosphorylation of a non-paired helical filament pool in Alzheimer disease. J. Biol. Chem. 268, 24374-24384.

Krstic, D., Pfister, S., Notter, T., Knuesel, I., 2013. Decisive role of Reelin signaling during early stages of Alzheimer's disease. Neuroscience 246, 108-116.

Lambert, M.P., Viola, K.L., Chromy, B.A., Chang, L., Morgan, T.E., Yu, J., et al., 2001. Vaccination with soluble Abeta oligomers generates toxicity-neutralizing antibodies. J. Neurochem. 79, 595-605.

Lane-Donovan, C., Philips, G.T., Wasser, C.R., Durakoglugil, M.S., Masiulis, I., Upadhaya, A., et al., 2015. Reelin protects against amyloid beta toxicity in vivo. Sci. Signal. 8, ra67.

Li, C., Gotz, J., 2017. Somatodendritic accumulation of Tau in Alzheimer's disease is promoted by Fyn-mediated local protein translation. EMBO J. 36, 3120-3138.

Lim, F., Hernandez, F., Lucas, J.J., Gomez-Ramos, P., Moran, M.A., Avila, J., 2001. FTDP17 mutations in tau transgenic mice provoke lysosomal abnormalities and Tau filaments in forebrain. Mol. Cell. Neurosci. 18, 702-714.

Lucas, J., Hernández, F., Gómez-Ramos, P., Morán, M., Hen, R., Avila, J., 2001. Decreased nuclear beta-catenin, tau hyperphosphorylation and neurodegeneration in GSK-3beta conditional transgenic mice. EMBO J. 20, 27-39.

Madronal, N., Delgado-Garcia, J.M., Gruart, A., 2007. Differential effects of long-term potentiation evoked at the CA3 CA1 synapse before, during, and after the acquisition of classical eyeblink conditioning in behaving mice. J. Neurosci. 27, 12139-12146.

Madronal, N., Gruart, A., Delgado-Garcia, J.M., 2009. Differing presynaptic contributions to LTP and associative learning in behaving mice. Front. Behav. Neurosci. 3, 7.

Ohkubo, N., Lee, Y.D., Morishima, A., Terashima, T., Kikkawa, S., Tohyama, M., et al., 2003. Apolipoprotein E and Reelin ligands modulate tau phosphorylation through an apolipoprotein E receptor/disabled-1/glycogen synthase kinase-3beta cascade. FASEB J. 17, 295-297.

Pujadas, L., Gruart, A., Bosch, C., Delgado, L., Teixeira, C.M., Rossi, D., et al., 2010. Reelin regulates postnatal neurogenesis and enhances spine hypertrophy and long-term potentiation. J. Neurosci. 30, 4636-4649.

Pujadas, L., Rossi, D., Andres, R., Teixeira, C.M., Serra-Vidal, B., Parcerisas, A., et al., 2014. Reelin delays amyloid-beta fibril formation and rescues cognitive deficits in a model of Alzheimer's disease. Nat. Commun. 5, 3443.

Reitz, C., Mayeux, R., 2014. Alzheimer disease: epidemiology, diagnostic criteria, risk factors and biomarkers. Biochem. Pharmacol. 88, 640-651.

Rice, D.S., Curran, T., 2001. Role of the reelin signaling pathway in central nervous system development. Annu. Rev. Neurosci. 24, 1005-1039.

Roberson, E.D., Scearce-Levie, K., Palop, J.J., Yan, F., Cheng, I.H., Wu, T., et al., 2007. Reducing endogenous tau ameliorates amyloid beta-induced deficits in an Alzheimer's disease mouse model. Science 316, 750-754.

Roberson, E.D., Halabisky, B., Yoo, J.W., Yao, J., Chin, J., Yan, F., et al., 2011. Amyloidbeta/Fyn-induced synaptic, network, and cognitive impairments depend on tau levels in multiple mouse models of Alzheimer's disease. J. Neurosci. 31, 700-711.

Selkoe, D.J., 2002. Alzheimer's disease is a synaptic failure. Science 298, 789-791.

Selkoe, D.J., 2008. Soluble oligomers of the amyloid beta-protein impair synaptic plasticity and behavior. Behav. Brain Res. 192, 106-113.

Shi, Y., Yamada, K., Liddelow, S.A., Smith, S.T., Zhao, L., Luo, W., et al., 2017. ApoE4 markedly exacerbates tau-mediated neurodegeneration in a mouse model of tauopathy. Nature 549, 523-527.

Sivanesan, S., Tan, A., Rajadas, J., 2013. Pathogenesis of Abeta oligomers in synaptic failure. Curr. Alzheimer Res. 10, 316-323.

Sohn, P.D., Tracy, T.E., Son, H.I., Zhou, Y., Leite, R.E., Miller, B.L., et al., 2016. Acetylated tau destabilizes the cytoskeleton in the axon initial segment and is mislocalized to the somatodendritic compartment. Mol. Neurodegener. 11, 47.

Soriano, E., Del Rio, J.A., 2005. The cells of cajal-retzius: still a mystery one century after. Neuron 46, 389-394.

Soto, C., Pritzkow, S., 2018. Protein misfolding, aggregation, and conformational strains in neurodegenerative diseases. Nat. Neurosci. 21, 1332-1340.

Spires-Jones, T., Knafo, S., 2012. Spines, plasticity, and cognition in Alzheimer's model mice. Neural Plast. 2012, 319836.

Walsh, D.M., Klyubin, I., Fadeeva, J.V., Cullen, W.K., Anwyl, R., Wolfe, M.S., et al., 2002. Naturally secreted oligomers of amyloid beta protein potently inhibit hippocampal long-term potentiation in vivo. Nature 416, 535-539.

Wang, Y., Mandelkow, E., 2016. Tau in physiology and pathology. Nat. Rev. Neurosci. 17, $5-21$.

Zempel, H., Thies, E., Mandelkow, E., Mandelkow, E.M., 2010. Abeta oligomers cause localized $\mathrm{Ca}(2+)$ elevation, missorting of endogenous Tau into dendrites, Tau phosphorylation, and destruction of microtubules and spines. J. Neurosci. 30, 11938-11950.

Zempel, H., Luedtke, J., Kumar, Y., Biernat, J., Dawson, H., Mandelkow, E., et al., 2013. Amyloid-beta oligomers induce synaptic damage via Tau-dependent microtubule severing by TTLL6 and spastin. EMBO J. 32, 2920-2937.

Zempel, H., Dennissen, F.J.A., Kumar, Y., Luedtke, J., Biernat, J., Mandelkow, E.M., et al., 2017. Axodendritic sorting and pathological missorting of Tau are isoform-specific and determined by axon initial segment architecture. J. Biol. Chem. 292, 12192-12207.

Zucker, R.S., Regehr, W.G., 2002. Short-term synaptic plasticity. Annu. Rev. Physiol. 64, $355-405$. 